Purdue University

Purdue e-Pubs

Open Access Theses

Theses and Dissertations

$12-2016$

\title{
Trajectory optimization using indirect methods and parametric scramjet cycle analysis
}

Joseph Williams

Purdue University

Follow this and additional works at: https://docs.lib.purdue.edu/open_access_theses

Part of the Aerospace Engineering Commons

\section{Recommended Citation}

Williams, Joseph, "Trajectory optimization using indirect methods and parametric scramjet cycle analysis" (2016). Open Access Theses. 908.

https://docs.lib.purdue.edu/open_access_theses/908

This document has been made available through Purdue e-Pubs, a service of the Purdue University Libraries. Please contact epubs@purdue.edu for additional information. 


\section{PURDUE UNIVERSITY \\ GRADUATE SCHOOL \\ Thesis/Dissertation Acceptance}

This is to certify that the thesis/dissertation prepared

By _Joseph Williams

Entitled

Trajectory Optimization Using Indirect Methods and Parametric Scramjet Cycle Analysis

For the degree of Master of Science in Aeronautics and Astronautics

Is approved by the final examining committee:

Michael Grant

Chair

William Crossley

Stephen Heister

To the best of my knowledge and as understood by the student in the Thesis/Dissertation Agreement, Publication Delay, and Certification Disclaimer (Graduate School Form 32), this thesis/dissertation adheres to the provisions of Purdue University's "Policy of Integrity in Research" and the use of copyright material.

Approved by Major Professor(s): Michael Grant

Approved by: Weinong Wayne Chen 12/7/2016 



\title{
TRAJECTORY OPTIMIZATION USING INDIRECT METHODS AND PARAMETRIC SCRAMJET CYCLE ANALYSIS
}

\author{
A Thesis \\ Submitted to the Faculty \\ of \\ Purdue University \\ by \\ Joseph Williams \\ In Partial Fulfillment of the \\ Requirements for the Degree \\ of \\ Master of Science in Aeronautics \& Astronautics
}

December 2016

Purdue University

West Lafayette, Indiana 


\section{ACKNOWLEDGMENTS}

First I would like to offer my sincerest appreciation to my adviser Professor Michael Grant. The opportunity he provided me in his lab has changed my approach to problems and research. Through his patience and guidance I am a far more capable engineer than before my studies at Purdue. I would also like to thank Professor William Crossley and Professor Stephen Heister for serving on my committee and reviewing my thesis.

To my research group, especially Thomas Anthony, Kshitij Mall, Harish Saranathan, and Zhenbo Wang, I learned a great amount from our interactions and collaborative work.

Finally, I must express my gratitude to my parents and to my wife for providing me with constant support and encouragement throughout my years of study. This thesis would not have been possible without them. 
TABLE OF CONTENTS

Page

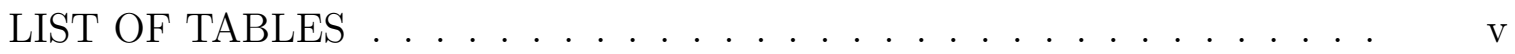

LIST OF FIGURES .......................... vi vi

SYMBOLS ....................................... vii

ABBREVIATIONS ......................

ABSTRACT ............................. xi

1 INTRODUCTION . . . . . . . . . . . . . . . . . . . 1

1.1 Motivation . . . . . . . . . . . . . . . . 1

1.2 History of Hypersonic Vehicles . . . . . . . . . . . . . . . . . . . 2

1.3 Previous Work in Trajectory Optimization . . . . . . . . . . 5

2 OVERVIEW OF INDIRECT TRAJECTORY OPTIMIZATION . . . . . 12

2.1 Calculus of Variations . . . . . . . . . . . . . . . . . . . 12

2.2 Necessary Conditions of Optimality . . . . . . . . . . . . . . . 13

2.3 Numerical Method . . . . . . . . . . . . . . . . . . . . . . 14

2.4 Challenges of Indirect Methods . . . . . . . . . . . . . . . . 14

3 EPSILON-TRIG REGULARIZATION . . . . . . . . . . . . . . . 16

3.1 Epsilon-Trig Regularization of Bang-Bang Control Problems . . . . 16

4 PROBLEM FORMULATION . . . . . . . . . . . . . . . . . . . . . . . . . . . . . . . . .

4.1 Vehicle Characterization . . . . . . . . . . . . . 20

4.1 .1 Aerodynamics . . . . . . . . . . . . . . . . 21

4.2 Equations of Motion and Coordinate System . . . . . . . . . . . . 22

4.3 Objective and Constraints . . . . . . . . . . . . 25

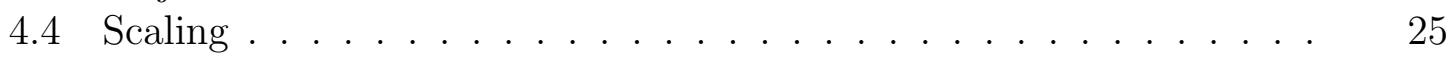

5 THE ROCKET PROBLEM . . . . . . . . . . . . . . 26

5.1 New Control . . . . . . . . . . . . . . . 26

5.1 .1 Justification . . . . . . . . . . . . . . . . . . . . . . 26

5.1.2 New Control Explained . . . . . . . . . . . . . . . . 27

5.2 Example Problem . . . . . . . . . . . . . . . . 28

6 THE SCRAMJET PROBLEM . . . . . . . . . . . . . . . . . . . . . . . . . . . . . . . . . . . . . .

6.1 The Thrust Model . . . . . . . . . . . . . . . . . 33

6.2 Scramjet Engine Bounding . . . . . . . . . . . . . . . . . . . . 35

6.3 Scramjet Solutions . . . . . . . . . . . . . . 36 
6.3.1 Epsilon-Trig Adjoining . . . . . . . . . . . . . . . . . . 44

6.3.2 Continuation Process . . . . . . . . . . . . 46

7 TRADE STUDIES . . . . . . . . . . . . . . . . . . . . 49

8 SUMMARY . . . . . . . . . . . . . . . . . . . . . 59

9 FUTURE WORK . . . . . . . . . . . . . . . . . . . 60

LIST OF REFERENCES . . . . . . . . . . . . . . . . . . . 62 


\section{LIST OF TABLES}

Table

4.1 Vehicle characteristics. . . . . . . . . . . . . . . . . . 20

4.2 Coefficients for the aerodynamic model. . . . . . . . . . . . . . . . . 22

4.3 Constants for the exponential atmosphere model. . . . . . . . . . . . . 24

5.1 Constants for the rocket missile problem. . . . . . . . . . . . . . . 29

5.2 Initial and final conditions for rocket missile problem. . . . . . . . . . . 30

5.3 Solver Performance. . . . . . . . . . . . . . . . . . . 32

6.1 Values for scramjet engine input. . . . . . . . . . . . . . . 40

6.2 Initial and final conditions for scramjet missile problem. . . . . . . . . 40

6.3 Solver Performance. . . . . . . . . . . . . . . . . . . 45

7.1 Values for scramjet engine input. . . . . . . . . . . . . . . . 49

7.2 Values for scramjet engine input. . . . . . . . . . . . . 51

7.3 Values for scramjet engine input. . . . . . . . . . . . . 54

7.4 Values for scramjet engine input. . . . . . . . . . . . . 56 


\section{LIST OF FIGURES}

Figure $\quad$ Page

1.1 X-43A Vehicle Schematic NASA LaRC Archive . . . . . . . . . . . . . 4

1.2 GDE-2 engine in NASA-LaRC. . . . . . . . . . . . . . . . 5

1.3 Accurate vehicle thrust vector $[36] \ldots \ldots \ldots$. . . . . . . . . 8

4.1 Aerodynamic model at varying Mach numbers. . . . . . . . . . . . . . 21

4.2 Curve fit aerodynamic model. . . . . . . . . . . . . . . . 22

5.1 State histories using bvp4c and GPOPS-II. . . . . . . . . . . . 31

5.2 Control histories using bvp4c and GPOPS-II. . . . . . . . . . . . 31

6.1 Range of scramjet engine model performance at $26 \mathrm{~km}$ altitude. . . . . 35

6.2 Scramjet engine operation limited by dynamic pressure. . . . . . . . . 36

6.3 State histories using GPOPS-II and BVP4C. . . . . . . . . . . . . . 42

6.4 Control histories using GPOPS-II and BVP4C. . . . . . . . . . 43

6.5 Dynamic pressure and Mach histories over the trajectory. . . . . . . . . 43

6.6 Costate histories explaining numerical spikes in controls. . . . . . . . . 45

6.7 Control accuracy given adjoining methodology. . . . . . . . . . . . 46

7.1 Altitude history with changing $T_{\text {Max }}$ values. . . . . . . . . . . 50

7.2 Mass by downrange study with changing $T_{\text {Max }}$ values. . . . . . . . . 50

7.3 Energy plot with changing $T_{\text {Max }}$ values. . . . . . . . . . . . . . 51

7.4 Altitude history with changing $A_{\text {Max }}$ values. . . . . . . . . . . . . . 52

7.5 Mass by downrange with changing $A_{\text {Max }}$ values. . . . . . . . . . . . . . 52

7.6 Energy plot with changing $A_{\text {Max }}$ values. . . . . . . . . . . . . 53

7.7 Altitude history with changing $M_{c}$ values. . . . . . . . . . . . . 54

7.8 Mass by downrange with changing $M_{c}$ values. . . . . . . . . . . . 55

7.9 Energy plot with changing $M_{c}$ values. . . . . . . . . . . . . 55

7.10 Altitude history with changing $h_{p r}$ values. . . . . . . . . . . . 56 
Figure $\quad$ Page

7.11 Mass by downrange with changing $h_{p r}$ values. . . . . . . . . . . . . 57

7.12 Energy plot with changing $h_{p r}$ values. . . . . . . . . . . . . . 57 


\section{SYMBOLS}

$C_{D} \quad$ Coefficient of drag

$C_{L} \quad$ Coefficient of lift

$D \quad$ Drag force magnitude, $\mathrm{N}$

$L \quad$ Lift force magnitude, $\mathrm{N}$

$M_{\mathrm{c}} \quad$ Combustion Mach number

$M_{0} \quad$ Freestream Mach number

T Thrust, $\mathrm{N}$

$T_{0} \quad$ Freestream temperature, $\mathrm{K}$

$T_{\text {Max }} \quad$ Static temperature at combustor exit, $\mathrm{K}$

$T_{\text {Max }}^{\prime} \quad$ Total temperature at combustor exit, $\mathrm{K}$

$f \quad$ Fuel-to-air ratio

$F / \dot{m}_{0} \quad$ Specific thrust, $\mathrm{m} / \mathrm{s}$

$h_{\mathrm{pr}} \quad$ Fuel heating value, $\mathrm{J} / \mathrm{kg}$

Isp Specific impulse, $\mathrm{s}$

$\gamma_{r} \quad$ Ratio of specific heats

$\tau_{\lambda} \quad$ Burner exit total temperature to freestream temperature ratio

$\tau_{r} \quad$ Inlet temperature ratio

$u_{\epsilon} \quad$ Error controls

$u_{\text {TRIG }}$ Trigonometric control

$V_{0} \quad$ Freestream velocity

$\epsilon \quad$ Error parameter

$\frac{L}{D} \quad$ Lift-to-drag ratio

$S_{\text {ref }} \quad$ Reference area, $\mathrm{m}^{2}$

$m \quad$ Mass, $\mathrm{kg}$

$q$ Dynamic pressure, $\mathrm{N} / \mathrm{m}^{2}$ 
$h_{\text {scale }} \quad$ Scale height, $\mathrm{m}$

$R_{E} \quad$ Radius of the Earth, $\mathrm{m}$

$r_{n} \quad$ Nose radius, $\mathrm{m}$

$t_{f} \quad$ Time of flight, $\mathrm{s}$

$H \quad$ Hamiltonian

$J \quad$ Cost function or Jacobian matrix

$V \quad$ Velocity, $\mathrm{m} / \mathrm{s}$

$\boldsymbol{x} \quad$ State vector

$\alpha \quad$ Angle of attack, rad

$\beta \quad$ Ballistic coefficient, $\mathrm{kg} / \mathrm{m}^{2}$

$\gamma \quad$ Flight path angle, rad

$\theta \quad$ Longitude, rad

$\boldsymbol{\lambda}$ Costate vector

$\mu \quad$ Standard gravitational parameter, $\mathrm{m}^{3} / \mathrm{s}^{2}$

$\rho \quad$ Density of the atmosphere, $\mathrm{kg} / \mathrm{m}^{3}$

$\rho_{0} \quad$ Atmospheric density at sea-level, $\mathrm{kg} / \mathrm{m}^{3}$ 


\begin{tabular}{ll} 
& \multicolumn{2}{c}{ ABBREVIATIONS } \\
BVP & Boundary Value Problem \\
EOM & Equation of Motion \\
FPA & Flight Path Angle \\
GDE & Ground Demonstration Engine \\
GHAME & Generic Hypersonic Aerodynamic Model Example \\
GHV & Generic Hypersonic Vehicle \\
GPOPS & General Purpose OPtimal Control Software \\
HRE & Hypersonic Research Engine \\
JTOH & Joint Technology Office on Hypersonics \\
NASP & National Aerospace Plane \\
NLP & Non-linear Programming \\
OCP & Optimal Control Problem \\
PSOC & Pseudo-Spectral Optimal Control \\
MPBVP & Multi-Point Boundary Value Problem \\
TPBVP & Two-Point Boundary Value Problem
\end{tabular}




\begin{abstract}
Williams, Joseph M.S.A.A., Purdue University, December 2016. Trajectory Optimization using Indirect Methods and Parametric Scramjet Cycle Analysis. Major Professor: Michael J. Grant.

This study investigates the solution of time sensitive regional strike trajectories for hypersonic missiles. This minimum time trajectory is suspected to be best performed by scramjet powered hypersonic missiles which creates strong coupled interaction between the flight dynamics and the performance of the engine. Comprehensive engine models are necessary to gain better insight into scramjet propulsion. Separately, robust and comprehensive trajectory analysis provides references for vehicles to fly along. However, additional observation and understanding is obtained by integrating the propulsion model inside the trajectory framework. Going beyond curve fitted thrusting models, an integrated scramjet cycle analysis offers rapid trade studies on engine parameters and enables the identification of the most significant and optimal engine parameters for the mission as a whole. Regularization of bang-bang control problems by use of the Epsilon-Trig regularization method has created the possibility to preserve the original equations of motion while still solving these problems through indirect methods. Indirect methods incorporate mathematical information from the optimal control problem to provide high quality, integrated solutions. The minimum time optimal trajectory of a rocket propelled missile is compared to that of a scramjet powered missile to determine the advantages of scramjet technology in this application.
\end{abstract}




\section{INTRODUCTION}

\subsection{Motivation}

The large velocities associated with scramjet powered systems make them appealing for use as take-off vehicles meant for orbital flight, long distance quick civilian transport, and for a wide range of military applications. The first and second offset strategies, nuclear weapons and stealth technology, have forced opponents to outspend the United States in hopes of defending against the new capability. This has provided the US with both a technological advantage and a systematic and cost advantage. With stealth fighter and bomber technology aging, the need for a third offset is necessary. This third offset may include the use of hypersonic technologies [38]. Scramjet engines provide the capability to fly air-breathing machines with a larger efficiency and potentially an expanded performance compared to its rocket and ramjet counterparts.

One of the main drivers for the development of hypersonic scramjet technology is its application in missile vehicles for time sensitive regional strike missions. From the Joint Technology Office on Hypersonics (JTOH) roadmaps, hypersonic weapons with a nominal range of 600-1000 nautical miles capable of precision engagement of high payoff, time sensitive, fixed/relocatable, moving, and deeply buried targets are desired to improve the performance of time sensitive regional strike missions [2]. On the Technology Product Roadmap, an expendable fuel cooled scramjet engine capable of extended flight at Mach 6 or greater is a prime candidate to fulfill this need.

Establishing a desired trajectory by controlling the attitude and propulsion commands impacts the performance of any aerospace vehicle. Designing trajectories for hypersonic scramjet vehicles is difficult due to the highly coupled nature of the propulsion system and the flight dynamics. This complicates modeling and can make it 
unclear how certain vehicle shape, propulsion, or trajectory parameters affect the performance of the system and mission overall. Understanding the optimal trajectories in which these prompt strike vehicles fly and knowing the effect specific engine variables have on the overall mission will provide insight into which parameters matter most and how to design and build more effective and efficient weapons.

Currently engine models in trajectory optimization rely on tabulated data from independently run advanced models and test data. Stepping away from the tabulated based models and towards integrated analytic thrust models within the solver's framework allows for more readily conducted trade studies on engine parameters with regards to the overall vehicle performance in terms of specific missions. This can aid program managers in focusing on specific designs and investing in research of particular components, ultimately creating a more efficient and better performing final product.

\subsection{History of Hypersonic Vehicles}

The first person to recognize the possibility of ram pressure as a means of propulsion was probably René Lorin in 1913. By 1928, a patent was issued for a propulsion device containing all the parts of a modern ramjet. Soon after, René Leduc received a patent for an airplane powered by ramjet technology. However, because of the Second World War, ramjet technology did not fly until 1949, reaching Mach 0.84 at 26,000 feet. This was the beginning of ramjet technology that would soon lead to scramjet propulsion and its application in missiles and vehicles.

In a ramjet, air reaches the engine inlet at supersonic speeds. Ramjets are designed to take this flow and decelerate it until it is subsonic for combustion before ejecting it back at supersonic speeds. As the flight Mach number increases, it becomes less efficient to decelerate the breathed air to subsonic velocities for combustion. This realization is what funded work on scramjet propulsion systems and more research airplanes. NASA has devoted several X-planes to the study of hypersonic flight. 
The Hypersonic Research Engine (HRE) Project started in 1964 was created with the goal of designing, developing, and constructing a hypersonic research engine that used ramjet or scramjet technology. As the engine was being developed, HRE paired up with the more senior X-15 experimental rocket program to use an X-15 vehicle that crashed in 1962 . The X-15 was rebuilt with modifications, adding space for a ramjet. The overhauled X-15, called X-15A-2, flew several times with an inactive ramjet and reached hypersonic speeds. Eventually the program was canceled in part due to excessive costs. However, more X-planes were to follow.

With the goal of single stage to orbit missions the National Aerospace Plane (NASP) program was started. This plane, designated the X-30, was meant to take off horizontally from a runway, travel through the atmosphere experiencing varying flight conditions and fly at Mach numbers as high as Mach 25. From a structural and aerodynamic standpoint, the design involved a highly integrated configuration of airframe and engine. This setup, referred to as a waverider, is an efficient body for high speed flight due to the compression lift, where the aircraft forebody acts as compression ramps to gather air from the sparse atmosphere for combustion, while the aftbody behaves as a nozzle. While the X-30 was canceled in 1993, a significant amount of aero-thermo-elastic-propulsion research was accomplished because of it. At the end, the scramjet component of the vehicle was never tested, but hypersonic flight research continued.

Announced in 1996, the Hyper-X (X-43) was a scaled down version of the X-30. At 12 feet and 2,800 pounds, the X-43 was designed to test the critical technologies remaining after the end of the X-30 program. The first flight test failed on June 2, 2001 due to lost control of the Pegasus booster after being deployed by a B-52. The second flight test was successful reaching Mach 6.83 and having controlled powered flight for 11 seconds at $24 \mathrm{~km}$. A third version of the X-43 flew 6 months later in November, 2004 reaching Mach 9.65 at $33.5 \mathrm{~km}$ and again breaking the speed record set from the second flight. 


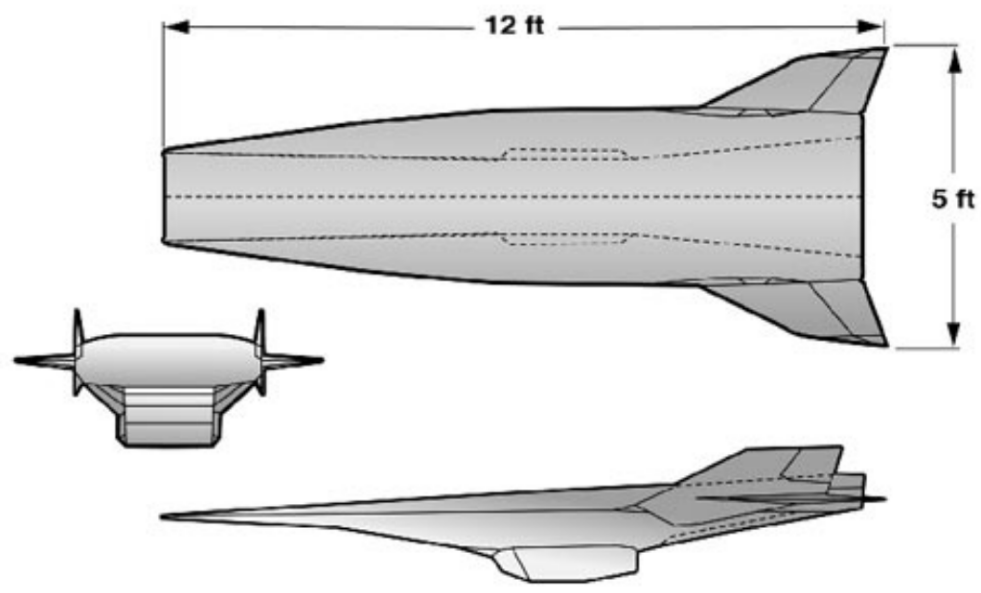

Figure 1.1. X-43A Vehicle Schematic NASA LaRC Archive

Another version of the X-43 was planned, the X-43C. Meant to be a slightly larger than the $\mathrm{X}-43 \mathrm{~A}$, the purpose of this version was to test vehicle cooling with its own hydrocarbon fuel. The built and tested X-43C scramjet engine also featured a variable geometry inlet compared to the fixed inlet of the X-43A. However, the X-43C was suspended indefinitely in March 2004.

The X-51A was similar to the successful X-43A. A joint Boeing and Pratt \& Whitney team began designing the Scramjet Engine Demonstrator that would be dropped by a B-52 near an altitude of $50 \mathrm{kft}$ and from there the 25 foot vehicle would be boosted to Mach 4.6 by a rocket. The goal was for the scramjet engine to ignite and propel the vehicle for 240 seconds, a vast improvemnt over the 11 seconds of the X-43A. [13] Extensive freejet testing was done on the first flight-weight hydrocarbonfueled scramjet in the early 2000's. Engineers performed more than 50 ground test attempts on the Gound Demonstration Engine 1 (GDE-1) using an open loop fuel source for cooling. Ground Demonstration Engine 2 (GDE-2) completed over 300 seconds of combustion with a closed loop fuel source for cooling at Mach 5. The first X-51 took flight on May 25, 2010. Despite the flight only lasting just over 200 seconds 
and having a scramjet burn time of 140 seconds, the experiment was considered a success. Using JP-7 jet fuel, the vehicle accelerated from Mach 4.5 to Mach 5.

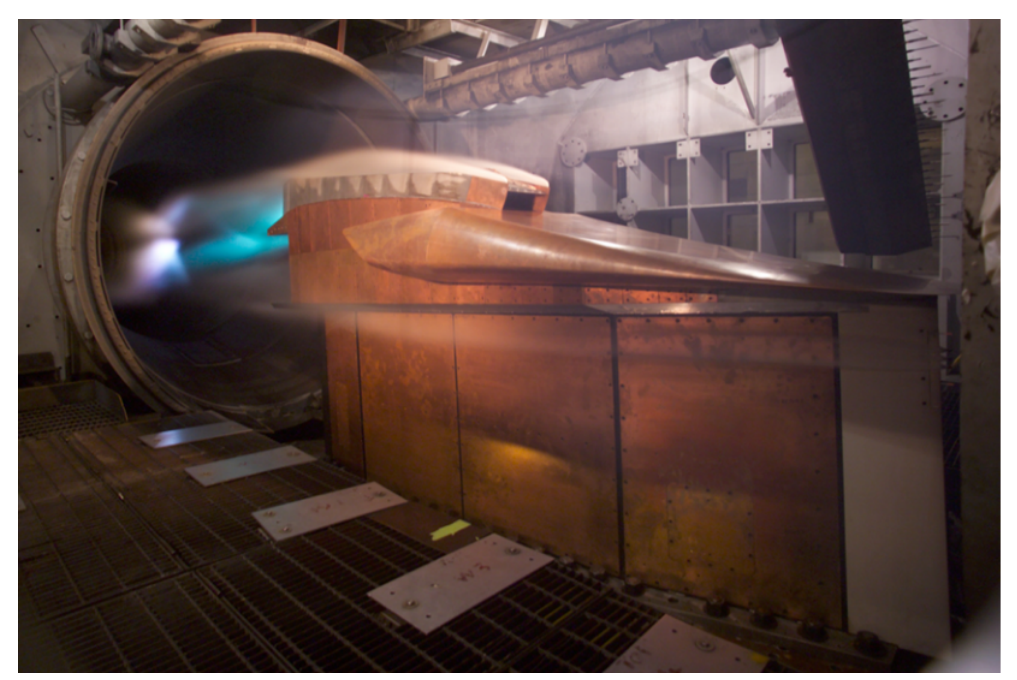

Figure 1.2. GDE-2 engine in NASA-LaRC.

The continued reduction in maximum altitude and Mach number from the $\mathrm{X}-30$ to the X-41A, and finally the X-51A is a direct result of the difficulty of hypersonic problems. It indicates the need for improved vehicle design methods as well as carefully planned demonstrations and tests.

\subsection{Previous Work in Trajectory Optimization}

Historically, hypersonic thrusting problems have been solved through various approaches. Broadly these approaches are categorized under the names of direct and indirect methods.

The most common direct method used for solving trajectory optimization problems is pseudo-spectral optimal control (PSOC). GPOPS and DIDO are two popular direct method solvers that utilize PSOC. The direct method arose with the expanding use of computers and computational power as the trajectory is broken down into nodes, and the state values at the nodes are moved around until a locally optimal solution to the problem is found. In principle, a direct method computes the objective 
function value and compares this value for different solutions to find a minimum without explicitly deriving the necessary conditions of optimality. Direct method solvers typically have three major benefits. The problems are generally easy to set-up, they are quick to run, and can be robust to a wide range of initial guesses.

There are different solution methods (some of which are much older than the direct methods) that have only started being utilized in trjaectory optimization in recent years. Indirect methods [11] offer the advantage of leveraging mathematical information present in the problem to guarantee locally optimal solutions. The benefits and difficulties of the indirect method will be discussed in more detail in Chapter 2.

There are several approaches to modifying a problem to make it easier to solver numerically:

- A common technique to approach more complicated problems is to partition the trajectory. This involves making predetermined assumptions about what the most optimal solution will look like on the whole. A couple of examples of this approach are seen in Ref. 3 and Ref. 4 and are discussed later in this chapter.

- Another approach to obtain better solutions for thrusting problems is to reduce the accuracy of the equations of motion. This method is particularly useful with indirect solvers and is seen in Ref. 5 .

- Rather than simplifiying the equations of motion as seen above when using an indriect method, another approach is to solve the control law using numerical methods [11].

- For air breathing problems, the usual strategy is to take data tables from tests or advanced models and form equations describing thrust from them. In these cases the engine model is only defined for the configurations used in the tests, limiting their applications in design studies that sweep a wide range of conditions. 
Addressing the first bullet, it is common to see two methods of partitioning trajectories into different phases. The first one has a climbing or ascent phase, followed by cruise, and finally descent. Another flight profile has a full thrust phase until propellant is expended, followed by a glide phase.

In Ref. 3, GPOPS was used to optimize a trajectory of an air-dropped and rocketboosted scramjet missile to maximize downrange. The trajectory was divided into three phases: ascent, cruise, and descent, and the beginning and end of each phase was predetermined. The cruise phase maintained an altitude of $20 \mathrm{~km}$ and a velocity of $2 \mathrm{~km} / \mathrm{s}$.

Another example of user selected trajectory architectures can be seen in Ref. 4. A fuel-optimal trajectory is split into two problems: an ascent and a maximum glide problem and then the two trajectories are joined to obtain the flight path. The benefit is that after the ascent phase, there is only one control, angle of attack, and the mass does not change.

Indirect solvers with analytically derived control laws have optimized boost glide trajectories for maximum downrange, where a rocket was given the ability to throttle on and off until the propellant mass was expended [5]. The solution method, similar to the one used in this work, requires derivatives with respect to control. Using angle of attack as control leads to the problem of transcendental equations and thus prevents a solution. Therefore, the angle of attack is assumed to be a small angle and the equations of motion are modified to remove the trigonometric terms in $\alpha$. This modification means that the thrust vector is assumed to be in the same direction as the wind or velocity vector. This can cause large errors when either $\alpha$ or the value of thrust is large. Fig. 1.3 highlights the fidelity that is lost throughout the entire powered portion of the trajectory by making a small angle approximation for $\alpha$.

In Ref. 11, a minimum fuel orbital ascent by a hypersonic air-breathing vehicle is investigated using optimal control theory. Two controls, angle of attack and throttle are used. The control law is solved with numerical methods instead of finding a closed 


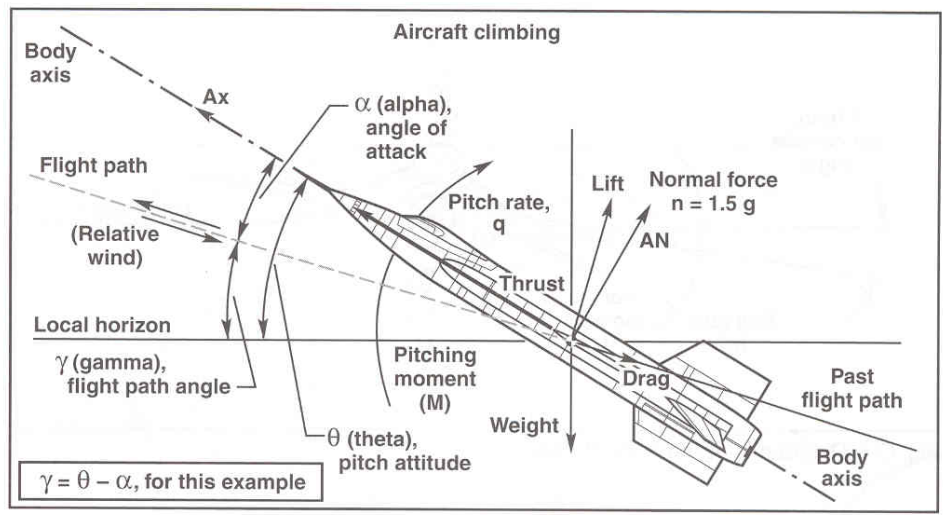

Fig. D-1. Diagram showing longitudinal terms with aircraft climbing.

Figure 1.3. Accurate vehicle thrust vector [36]

form, analytic solution. Additionally constraints were placed on both angle of attack and the dynamic pressure in which the vehicle could operate.

Commonly, in trajectory optimization of an air breathing hypersonic vehicle, the propulsion model is defined as curve fits from more complicated computer simulations or experimental data that have been tabulated. The thrust models previously used in hypersonic trajectory problems typically originate from Eq. (1.1), where $I_{s p}$ is the specific impulse, $\dot{m}_{f}$ is the mass flow rate of fuel, and $g_{0}$ is the gravity as sea level.

The thrust equation:

$$
T=\dot{m}_{f} g_{0} I_{s p}
$$

An example of engine curve fitting for thrust models in trajectory optimization can be found in Ref. 3. Specific impulse and air intake rate, $\dot{m}_{a}$, are curve fit from data at $32.5 \mathrm{~km}$. Intake flow rate is used to calculate fuel-mass flow rate, which is then input into Eq. 1.1 to find thrust. This is represented in the following equations, where the engine control is the equivalence ratio, $\psi$. The equivalence ratio is the ratio of the actual fuel-air ratio and the fuel-air ratio that occurs when the combustion process consumes all oxygen. 


$$
\begin{array}{r}
I_{s p}=f(M, \alpha) \\
\dot{m}_{a}=f(M, \alpha) \text { at } 32.5 \mathrm{~km} \\
\dot{m}_{f}=f\left(\dot{m}_{a}, \psi\right)
\end{array}
$$

Other work uses a similar thrust model in which the thrust coefficient is curve fit and then is multiplied by the dynamic pressure [17]. In this case, the thrust coefficient and specific impulse are functions of the Mach number, dynamic pressure, and fuel-equivalence ratio.

A Generic Hypersonic Vehicle (GHV) model or Generic Hypersonic Aerodynamic Model Example (GHAME) are often used. In these models, the thrust coefficient and specific impulse are defined as functions of freestram Mach number and angle of attack (thrust coefficient) or throttle (specific impulse). [15]. Several engine cycles are run covering the prominent configurations for hypersonic flight and the stages to achieve that condition: turbojet, ramjet, and scramjet cycles. [16] The thrust model built from these components is derived from the thrust equation and can be seen below in Eq. (1.3). These models are useful in setting up problems involving powered flight trajectories. Developing the GHV engine model, the specific impulse becomes a curve fit function, and mass flow rate of fuel is reduced to its several components. The second of two curve fit functions is the coefficient of thrust. The results are seen in Eq. (1.3).

$$
T=0.5 \phi I_{s p}(\phi, M) \rho v g_{0} C_{T}(\alpha, M)
$$

The research presented in this thesis does not advance scramjet cycle analysis but rather attempts to determine if there are flight conditions being ignored that may be critical to mission success. It could direct research toward areas not being considered in current scramjet propulsion modeling research.

The development of scramjet modeling has been a research topic spanning many decades with large amounts of funding for testing and flight verification occuring fol- 
lowed by periods of little funding. Much of the work done on scramjets has been summarized in [18]. Inlet design issues include capturing the flow, starting the engine, viscous flow effects, and boundary-layer separation. Selecting wedge angles or an isentropic ramp to align the oblique shocks for proper air intake is a significant issue for high speed travel and the main purpose of the inlet. The combustion process is another critical component of the engine which has been studied in depth. Fuel injectors and air-fuel mixing are major considerations in combustion design and mixing techniques are widely studied. Finally combustion reactions with flow temperature increase and exit species are investigated.

In Ref. [19], the idea of expanding supersonic combustion to lower Mach numbers while maintaining performance at higher Mach number flight is considered. This work utilizes a stream thrust analysis to analyze the scramjet performance because of the many engine parameters that it involves. A common dynamic pressure value of $47,880 \frac{\mathrm{N}}{\mathrm{m}^{2}}$ is used and held constant throughout Roberts' work.

The idea of coupling research in engines and trajectories is not new. Dalle focused on integrating optimal engine design with optimal trajectories in Ref. [20]. This work optimized a hypersonic vehicle geometry and trajectory through detailed investigation of supersonic inlets and modeling of complex shock interactions, finite-rate chemistry, pre-combustion shock trains, and more. It should be noted that the approach Dalle took is opposite of the one presented in this work. Starting with the engine, the modeling of the scramjet is extensive, but the trajectory is designed by assuming a constant dynamic pressure and the vehicle's flight profile is restricted to consecutive attached lines of constant dynamic pressure. This is opposed to starting with the trajectory and building up the engine model once initially integrated.

Prior research has explored improving scramjet models and adding complexity to more accurately capture engine behavior. These works focus on better modeling the scramjet propulsion system with often greatly simplified trajectories. Since the focus is on the engine model, the trajectories considered are usually along a constant dynamic pressure line. Often the maximum dynamic pressure is chosen to maxi- 
mize the thrust of the vehicle. While these investigations are mostly looking at the performance of the engine at a common flight condition, it is important to know if mission design requires the engine to unstart/restart or if most of the operation is at an unexpected flight condition.

In this study, the two areas of engine modeling and trajectory optimization are bridged in a unique and more promising way through an indirect method of optimization. This guarantees a locally optimal solution while retaining the full accuracy of the equations of motion and without presupposing any phases in the trajectory as seen in previous works. Implementing an engine cycle analysis within trajectory optimization allows for engine parameter design and tuning analysis of the engine's overall effect on the mission. The scramjet thrusting model integrated into the solver is a parametric, ideal, thermodynamic cycle analysis that at this stage is not as complex as the work seen in Refs. [19] and [20]. The model can be developed to a more comprehensive state. 


\section{OVERVIEW OF INDIRECT TRAJECTORY OPTIMIZATION}

\subsection{Calculus of Variations}

Trajectory optimization problems are generally defined by a cost functional, $J$,

that is composed of a terminal cost, $\phi$ and a path cost, $\int_{t_{0}}^{t_{f}} L(x, u, t) \mathrm{d} t$. The optimization process will evaluate the cost functional and minimize it while satisfying the dynamics of the problem, $f$ as well as the initial and terminal boundary conditions, $\Psi$ and $\Phi$ respectively [33].

$$
\operatorname{Min} J=\phi\left(\boldsymbol{x}\left(t_{f}\right), t_{f}\right)+\int_{t_{0}}^{t_{f}} L(\boldsymbol{x}, \boldsymbol{u}, t) \mathrm{d} t
$$

Subject to :

$$
\begin{aligned}
& \dot{\boldsymbol{x}}=\boldsymbol{f}(\boldsymbol{x}, \boldsymbol{u}, t) \\
& \boldsymbol{\Psi}\left(\boldsymbol{x}\left(t_{0}\right), t_{0}\right)=0 \\
& \boldsymbol{\Phi}\left(\boldsymbol{x}\left(t_{f}\right), t_{f}\right)=0 \\
& t_{0}=0
\end{aligned}
$$

Trajectory optimization of powered hypersonic vehicles is complex due to the complex dynamics and coupled nature of the problem. There are mainly two classes of methods used to solve such problems, direct and indriect methods. The method used in this thesis is an indirect method. The main tool of the indirect method is calculus of variations, a field of mathematics that provides a framework to optimize functionals, such as $J$, and find their extrema. 


\subsection{Necessary Conditions of Optimality}

Indirect methods optimize the cost functional $J$ shown in Eq. (2.1) by formulating a multi-point boundary value problem that represents the necessary conditions of optimality. In this sense, the problem is first optimized by forming Eqs. (2.5a-2.5f) and then it is converted into a boundary value problem (BVP).

The necessary conditions of optimality are defined as follows:

$$
\begin{aligned}
& H=L(x, u, t)+\boldsymbol{\lambda}^{T}(t) \boldsymbol{f}(\boldsymbol{x}, \boldsymbol{u}, t) \\
& \dot{\boldsymbol{\lambda}}=-\frac{\partial H}{\partial \boldsymbol{x}} \\
& \frac{\partial H}{\partial \boldsymbol{u}}=0 \\
& \boldsymbol{\lambda}\left(t_{0}\right)=\boldsymbol{\nu}_{\mathbf{0}}^{T} \frac{\partial \boldsymbol{\Psi}}{\partial \boldsymbol{x}\left(t_{0}\right)} \\
& \boldsymbol{\lambda}\left(t_{f}\right)=\left(\frac{\partial \phi}{\partial \boldsymbol{x}\left(t_{f}\right)}+\boldsymbol{\nu}_{\boldsymbol{f}}^{T} \frac{\partial \boldsymbol{\Phi}}{\partial \boldsymbol{x}\left(t_{f}\right)}\right) \\
&\left(H+\frac{\partial \phi}{\partial t}+\boldsymbol{\nu}_{\boldsymbol{f}}^{T} \frac{\partial \boldsymbol{\Phi}}{\partial t}\right)_{t=t_{f}}=0
\end{aligned}
$$

Optimal control theory (OCT) introduces mathematical entities called costates for each dynamic state. Each has its own equation defined by Eq. (2.5b). The Hamiltonian is augmented with costates and Langrange Multipliers. Eq. (2.5a) defines the augmented Hamiltonian, where $\boldsymbol{\lambda}$ is the costate vector. Eqs. (2.5b-2.5f) describe the necessary conditions of optimality and form a well-defined BVP. The optimal control law, $\boldsymbol{u}(t)$ is obtained as a function of the states and costates by solving Eq. (2.5c). The initial and terminal boundary conditions on the costates are specified in Eqs. (2.5d) and (2.5e), where $\nu_{0}$ and $\nu_{f}$ are the Langrange Multipliers which are used to adjoin these boundary conditions to the cost functional. In addition to the boundary conditions, free parameters can exist in the optimization problem, such as time of flight. 


\subsection{Numerical Method}

As described above, OCT converts trajectory optimization problems into BVPs. The boundary conditions in the BVP can be root solved to obtain the optimal trajectory. The specific values for the initial conditions and free parameters need to be found that when propogated forward by integrating the equations of motion, satisfy the terminal boundary conditions. Several numerical solvers exist to solve such BVPs, including single shooting, multiple shooting, and collocation. Matlab offers an easy to use and reasonably robust, collocation solver called BVP4c which is used in this thesis [24].

\subsection{Challenges of Indirect Methods}

The indirect method provides explicit expressions for the optimal control as a function of the dynamic states and costates which guarantee a locally optimal trajectory. However, this method has challenges [23] described below, which are particularly relevant to hypersonic trajectory problems.

- The first difficulty is in formulating the boundary value problem for the given optimization problem. This requires detailed knowledge about the system, familiarity with optimal control theory, and a strong theoretical background.

- Path inequalities add another dimension of issues to solving the optimal control problem. It is necessary to know before hand how often the path constraints are active and the sequence in which they are active and not active.

- The third, well recognized obstacle is the sensitivity of the indirect method to the initial guess and its lack of robustness. Initial guesses of the states and especially co-states need to be very accurate for a solution be found. Co-states are mathematical entities with no physical meaning which makes it difficult to provide reasonable starting values. 
A technique called continuation is utilized to overcome the challenge of providing an accurate initial guess. The process begins by first solving for a simple trajectory. The problem is evolved through a series of steps into a desired problem. Solutions from previous steps are used as the initial guess for subsequent steps [39]. Additionally, modern symbolic math tools make it easier to formulate the BVPs automatically.

These techniques have successfully been used to solve optimal hypersonic trajectories. When the addition of powered flight is integrated into hypersonic problems and especially air-breathing powered flight, the dynamics of the problem become complex enough to prevent the creation of solutions using the techniques presented above. This work explores the strategies used to solve air-breathing powered flight trajectory problems using indirect methods and the challenges associated with it. 


\section{EPSILON-TRIG REGULARIZATION}

\subsection{Epsilon-Trig Regularization of Bang-Bang Control Problems}

A feature of optimal control problems (OCPs), detailed in Chapter 2, is the bangbang nature of certain bounded controls. When a bounded control appears in a linear form in the state equations, that control value will often stay at extremum values. At certain locations along the trajectory, a bang-bang control may change from one boundary to another. This switching from a minimum to maximum value and vice versa create numerical issues when using a gradient-based solution method like optimal control theory. Common bang-bang controls include throttle, which makes this issue relevant to the work presented in this thesis.

Techniques have been developed to overcome the gradient issue with bang-bang

controls. A smooth regularization technique was developed in [26], which introduced error controls, $u_{\epsilon_{n}}$, and an error parameter, $\epsilon$. Together the error parameters are able to smooth the transition from one boundary to the other. Dimensional consistency issues, as well as a lack of control bounding, in this method drove the founding of the next regularization technique.

A different regularization method was developed in [27] and expanded on in [28]. This method uses the concept of trigonomerization to express the control in the form of trigonometric functions, which implicitly bounds the control. When trigonomerization is applied upon bang-bang control problems, Eq. (3.1) is converted into Eq. (3.2), which contains control as a sine function.

$$
\begin{gathered}
H=H_{0}(t, x(t),(t))+H_{1}(t, x(t),(t)) u \\
H=H_{0}(t, \boldsymbol{x}(t), \boldsymbol{\lambda}(t))+H_{1}(t, \boldsymbol{x}(t), \boldsymbol{\lambda}(t)) \sin u_{\text {TRIG }}
\end{gathered}
$$


The Epsilon-Trig regularization method introduces a disturbance in the state equations, $\epsilon$, similar to the smoothing method, and creates an error control in trigonometric form. The error control is combined with its parameter, $\epsilon$. A major simplification over the previous method is that the reformulated OCP needs to have only one error control, $u_{\text {TRIG }}$, for each original control, keeping the new system of equations close to the original system. It should be noted that if there are two bang-bang controls then two separate error parameters are required to compliment the respective error controls.

Error parameters carry with them dimensions based on their complimentary control and the dynamic equation to which they are adjoined. Eq. (3.3) provides the unit of an individual $\epsilon$.

$$
\text { Unit of } \epsilon=\frac{\text { Unit of state equation }}{\text { Unit of complimentary control }}
$$

The method works by forming two orthogonal control components with the trigonometric functions. One component is the smooth control and the other is the error control. By increasing the error, the switching is smoothed.

The Epsilon-Trig regularization method is demonstrated below by reformulating the system of $\mathrm{k}+1$ equations in Eqs. (3.4) to the system seen in Eqs. (3.5). The objective function as shown in Eq. (3.4a) remains unchanged. Likewise, k-1 state equations where neither the error nor control appear remain unchanged. These equations are represented in Eq. (3.4c). For demonstration, the error is introduced into one state equation, Eq. (3.4b), and it is reformulated to a form shown in Eq. (3.5b). The state equation where the control appears originally in the form shown in Eq. (3.4d) is converted to a smooth trigonometric form as shown in Eq. (3.5d). The result is a smooth control and an error control in a trigonometric form such that Eq. (3.5e) is obtained. One trigonometric control, $u_{\mathrm{TRI}}$, can then be used to calculate both the smooth and the error controls. 
Minimize:

$$
J=J\left(t_{f}, x\left(t_{f}\right)\right)
$$

Subject to:

$$
\begin{aligned}
\dot{x}_{\epsilon} & =f_{1}(t, x(t)) \\
\dot{x}_{n} & =f_{n}(t, x(t)) \\
\dot{x}_{k+1} & =f_{k+1}(t, x(t))+u \\
2 & \leq n \leq k
\end{aligned}
$$

Minimize:

$$
J=J\left(t_{f}, x\left(t_{f}\right)\right)
$$

Subject to:

$$
\begin{aligned}
\dot{x}_{\epsilon} & =f_{1}(t, x(t))+\epsilon \cos u_{T R I G} \\
\dot{x}_{n} & =f_{n}(t, x(t)) \\
\dot{x}_{k+1} & =f_{k+1}(t, x(t))+\sin u_{T R I G} \\
\sin ^{2} u_{T R I G} & +\cos ^{2} u_{T R I G}=1 \\
2 & \leq n \leq k
\end{aligned}
$$

Using Eq. (2.5c) the optimal control options found are shown in Eqs. (3.6).

$$
u_{T R I G}^{*}=\left\{\begin{array}{l}
\arctan \left(\frac{\lambda_{x_{k+1}}}{\epsilon \lambda_{x_{1}}}\right) \\
\arctan \left(\frac{\lambda_{x_{k+1}}}{\epsilon \lambda_{x_{1}}}\right)+\pi
\end{array}\right.
$$

It is possible that the costate $\lambda_{x_{1}}$ in Eqs. (3.6) can vanish. This can again lead to additional numerical issues. Alternatively the nature of the OCP may mean that $\lambda_{x_{1}}$ 
in the optimal solution is zero leading to same problem. Therefore it is necessary to choose the adjoining dynamic equation with some thought to avoid these scenarios. The adjoining location will be addressed in Section 6.3.1. 


\section{PROBLEM FORMULATION}

\subsection{Vehicle Characterization}

Two hypersonic vehicle models are used in this study. The first is for the missile powered by the rocket and is defined in the works of [3] and [7]. The vehicle is a larger class missile that is capable of reaching distances farther than the maximum desired target distance adopted from the JSTO. However, the range discrepancy is sufficiently small that the vehicle can be used as a comparison for the scramjet powered missile. The second hypersonic vehicle model is a blended model drawing from the aforementioned works as well as [30]. Investigating [30], the hypersonic missile used is more suitable for a regional strike scramjet missile because of its size, however the available vehicle information is limited. Therefore, the size of the missile in weight is taken from [30] while the previous vehicle's aerodynamics were applied to this smaller missile. Additionally parameters like dry mass and reference area were scaled down to an appropriate value. It is expected that a larger missile will have a larger mass margin for propellant, therefore the dry to wet mass ratio of the scramjet missile is smaller. Both vehicle characteristics are summarized in Table 4.1.

Table 4.1. Vehicle characteristics.

\begin{tabular}{lcc} 
Vehicle Parameter & Rocket & Scramjet \\
\hline $\mathrm{S}_{\text {ref }}\left(\mathrm{m}^{2}\right)$ & 1 & 0.35 \\
\hline Wet Mass $(\mathrm{kg})$ & 3600 & 1300 \\
\hline Dry Mass $(\mathrm{kg})$ & 2000 & 600 \\
\hline Capture Area $\left(\mathrm{m}^{2}\right)$ & N.A. & 0.3 \\
\hline
\end{tabular}




\subsubsection{Aerodynamics}

The aerodynamic model in [7] used coefficients for lift and drag that varied with both angle of attack (AoA) and Mach number, $\left[C_{L}\left(M_{0}, \alpha\right), C_{D}\left(M_{0}, \alpha\right)\right]$. While incorporating this model into this thesis, the Mach number was assumed to be 6 . Inaccuracies, from the missing fidelity, were introduced from this decision, seen in Fig. 4.1. However, from comparing Eqs. (4.1a) and (4.1b), the model was greatly simplified which directly simplifies the control law. Note these equations input $\alpha$ in degrees. Future work can expand the aerodynamic model accuracy. The original model contained a limited range of AoA, starting at 0 degrees and ending at 8 degrees. While hypersonic vehicles have a relatively small operational range of AoA, this was considered too constrained for a design trade study [35]. Therefore the range of AoA was extended to -10 to 10 degrees as seen in Fig. 4.2. In the fitting, the coefficient of lift is assumed to be linear while the coefficient of drag is quadratic. The fittings are detailed in Eq. (4.2) and Table 4.2.
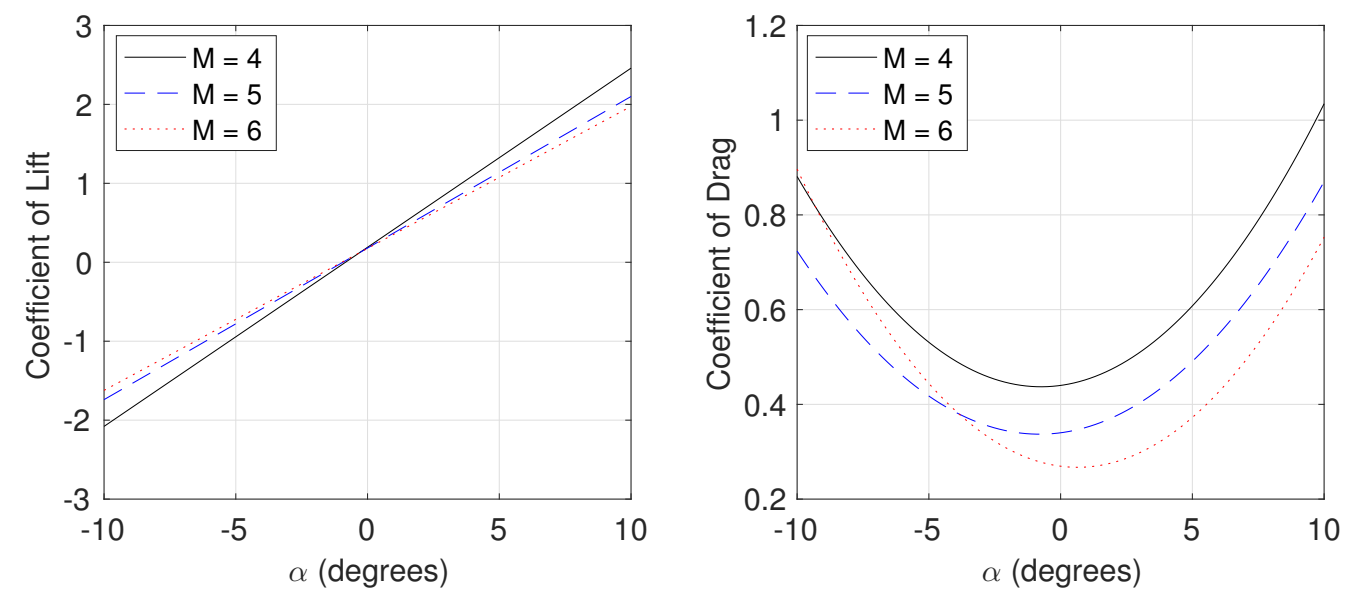

Figure 4.1. Aerodynamic model at varying Mach numbers. 

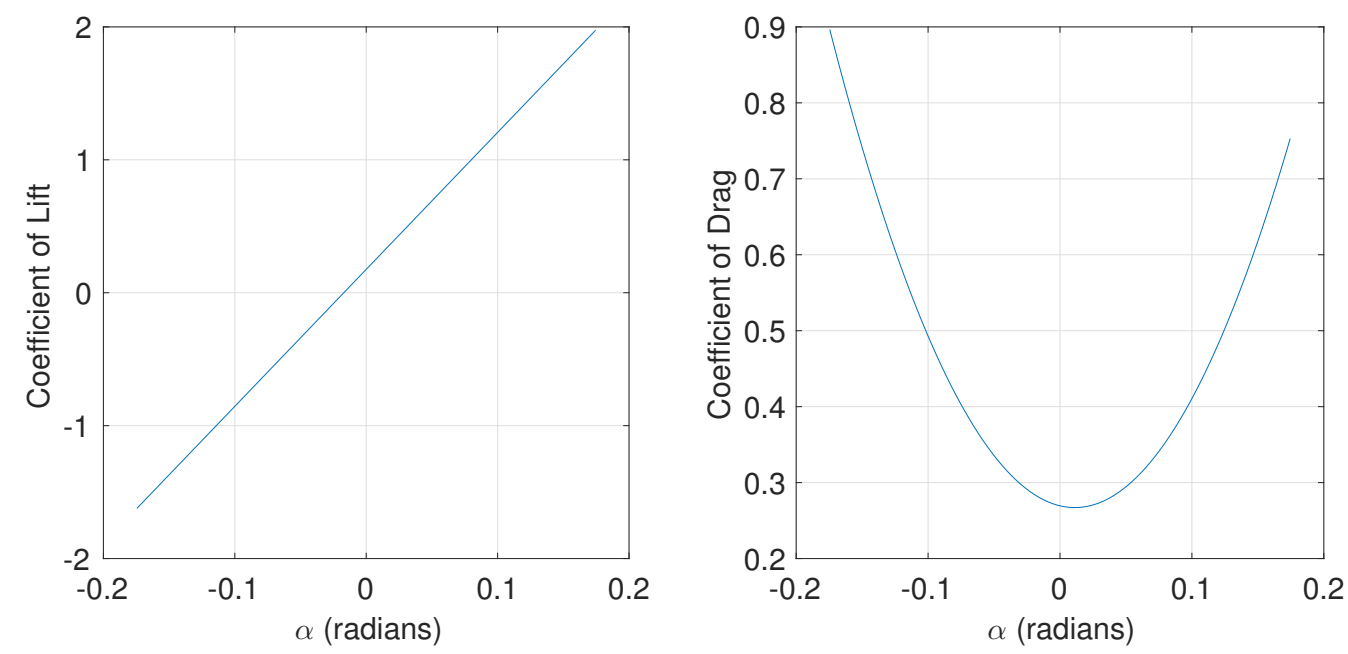

Figure 4.2. Curve fit aerodynamic model.

$$
\begin{gathered}
C_{L}=\left(-0.008 M^{3}+0.133 M^{2}-0.793 M+2.648\right)\left(0.001 \alpha^{2}+0.2 \alpha+0.19\right) \\
C_{L}(M=6)=0.00095 \alpha^{2}+0.19 \alpha+0.1805 \\
C_{L}(M=6, \alpha)=C_{L_{1}}(\alpha) \alpha+C_{L_{0}}(\alpha) \\
C_{D}(M=6, \alpha)=C_{D_{2}}(\alpha) \alpha^{2}+C_{D_{1}}(\alpha) \alpha+C_{D_{0}}(\alpha)
\end{gathered}
$$

Table 4.2. Coefficients for the aerodynamic model.

\begin{tabular}{lcccc}
$\mathbf{C}_{\mathbf{L}_{\mathbf{1}}}(\mathbf{1} / \mathrm{rad})$ & $\mathbf{C}_{\mathbf{L}_{\mathbf{0}}}$ & $\mathbf{C}_{\mathbf{D}_{\mathbf{2}}}\left(\mathbf{1} / \mathbf{r a d}^{2}\right)$ & $\mathbf{C}_{\mathbf{D}_{\mathbf{1}}}(\mathbf{1} / \mathbf{r a d})$ & $\mathbf{C}_{\mathbf{D}_{\mathbf{0}}}$ \\
\hline 10.305 & 0.1758 & 18.231 & -0.4113 & 0.26943 \\
\hline
\end{tabular}

\subsection{Equations of Motion and Coordinate System}

A vehicle-centric polar coordinate system and 2-DOF dynamic model are used to develop the equations of motion used in the present work. These can be seen in 
Eqs. (4.3). As is common, the states are altitude, downrange angle $(\theta)$, velocity, flight path angle $(\gamma)$, and vehicle mass [31]. Traditionally angle of attack, $(\alpha)$, is used as a control however here it is transitioned to a sixth state. The controls become rate of change of angle of attack, $\dot{\alpha}$, and thrust, $T$. These controls are converted to their trignometric form as explained in Chapter 3 and seen in Eqs. (4.3o) and (4.3p). A spherical Earth gravity model with an exponential atmosphere is assumed with the parameters shown in Table 4.3. The atmosphere model used makes an assumption of a constant temperature throughout the entire atmosphere. By using this atmospheric model, fidelity is lost when using engine models that utilize the inlet temperature for the cycle analysis. This is the case for the present work.

$$
\begin{aligned}
\dot{h} & =V \sin \gamma \\
\dot{\theta} & =\frac{V \cos \gamma}{r} \\
\dot{V} & =\frac{T \cos \alpha-D}{m}-\frac{\mu \sin \gamma}{r^{2}} \\
\dot{\gamma} & =\frac{(L+T \sin \alpha) \cos \sigma}{m V}+\left(\frac{V}{r}-\frac{\mu}{V r^{2}}\right) \cos \gamma \\
\dot{m} & =-\frac{T}{g_{0} I_{s p}} \\
\dot{\alpha} & =\alpha_{\text {rate }} \sin \left(\dot{\alpha}_{\text {TRIG }}\right)
\end{aligned}
$$


Where:

$$
\begin{aligned}
r & =R_{e}+h \\
\rho & =\rho_{0} e^{\frac{-h}{H_{\text {Scale }}}} \\
L & =q S_{r e f} C_{L} \\
C_{L} & =C_{L_{1}} \alpha+C_{L_{0}} \\
D & =q S_{r e f} C_{D} \\
C_{D} & =C_{D_{2}} \alpha^{2}+C_{D_{1}} \alpha+C_{D_{0}} \\
\phi & =\left(\frac{1+\sin T_{\mathrm{TRIG}}}{2}\right) \\
T & =T_{M A X} \phi \\
u_{1} & =\dot{\alpha}_{\mathrm{TRIG}} \\
u_{2} & =T_{\mathrm{TRIG}}
\end{aligned}
$$

In Table $4.3, R_{e}$ is the radius of the planet, Earth, $\mu$ is the gravitational parameter of the planet, $\rho_{0}$ is the surface atmospheric density of the planet, and $H_{\text {scale }}$ is the scale height of the exponential atmosphere model.

Table 4.3. Constants for the exponential atmosphere model.

\begin{tabular}{lc} 
Parameter & Value \\
\hline $\mathrm{R}_{\mathrm{e}}(\mathrm{km})$ & 6378 \\
\hline$\mu\left(\mathrm{m}^{3} / \mathrm{s}^{2}\right)$ & $3.986 \mathrm{e} 14$ \\
\hline$\rho_{0}\left(\mathrm{~kg} / \mathrm{m}^{3}\right)$ & 1.2 \\
\hline $\mathrm{H}_{\text {Scale }}(\mathrm{km})$ & 7.5 \\
\hline $\mathrm{g}_{0}\left(\mathrm{~m} / \mathrm{s}^{2}\right)$ & 9.81 \\
\hline
\end{tabular}




\subsection{Objective and Constraints}

The objective in a time sensitive trajectory is to reach the target as quickly as possible given a set of constraints. These constraints include: engine operational limits in both Mach number and dynamic pressure, target downrange location, limitations on $\dot{\alpha}$, and maximum possible fuel to burn. The vehicle is assumed to have been flown or boosted to an altitude and velocity conducive to scramjet engine operation. This is incorporated as initial state constraints on altitude, downrange, velocity, and mass. The target location and fuel capacity of the missile lead to terminal state constraints in altitude, downrange, and mass.

\subsection{Scaling}

Scaling is crucial to the convergence of the problem. Even with a simple planar problem, the dynamics are complex enough that without scaling no solution could be found. Scaling is used to bring all of the values of the states into the same order or nearly so. Distance, in this case altitude, time, and mass are all scaled at first by the initial values of the initial guess. Temperature is scaled by the constant maximum combustion temperature. These scaling parameters are updated throughout the continuation process by using the maximum value of the previous step. Note, time is scaled by Eq. (4.4).

$$
\text { time }(\mathrm{s})=\frac{\mathrm{h}(\mathrm{m})}{\mathrm{V}\left(\frac{\mathrm{m}}{\mathrm{s}}\right)}
$$




\section{THE ROCKET PROBLEM}

\subsection{New Control}

\subsubsection{Justification}

It has been discussed that the traditional control for hypersonic vehicles is angle of attack. In the context of optimal control theory, when this control is applied to a powered flight vehicle, solving for the control law results in a transcendental equation that makes it impossible to find the root solution of $\frac{d H}{d \alpha}=0$. The planar problem in Eq. (4.3), can be used to demonstrate this if the sixth state is removed and $\alpha$ is used as the control.

The Hamiltonian is formed from Eq. (2.5a). The derivative of the Hamiltonian with respect to the control results in the optimal control law for angle of attack seen in Eq. (5.1). The only contributing states to the control law are velocity and $\gamma$ :

$$
\frac{d H}{d \alpha}=\lambda_{V} \frac{-T \sin (\alpha)-\left(2 q S_{r e f} \alpha C_{D_{2}}+C_{D_{1}}\right)}{m}+\lambda_{\gamma} \frac{T \cos (\alpha)+q S_{r e f} C_{L_{1}}}{m V}=0
$$

It can be observed that the control law is transcendental because of the presence of polynomial and trigonometric expressions of control, $\alpha$. This means that a closed form analytic solution cannot be found. Other works have avoided this issue through various methods such as simplifying the equations of motion by assuming thrust is in the line with the drag force, reducing the trignometric expressions of $\alpha$ into polynomials, or by solving the control law numerically $[5,8,9]$. An alternative approach that preserves the equations of motion while still finding an analytic control law is taken here. 


\subsubsection{New Control Explained}

The original control, $\alpha$, is converted into a state variable and its time-derivative can be made the new control, u, as shown in Eq. (5.2).

$$
\dot{\alpha}=u
$$

The control is rewritten in a trigonometric form as shown in Eq. (4.3f) and following the discussion in Chapter 3. The second control, thrust, assumes that the rocket can be throttled between full thrust and no thrust as often as required by the optimal control problem. This control is also converted to trigonometric form in Eq. (4.3m). The error controls were adjoined to state equation corresponding to mass. The new equation of motion, parameters, and bounding variables are described in Eqs. (5.3a)- (5.3e).

$$
\begin{aligned}
& \dot{\alpha}=\alpha_{\mathrm{RATE}} \sin u_{\mathrm{TRIG}} \\
& \dot{m}=-\frac{T}{g_{0} I s p}+\epsilon_{1} \cos \left(\dot{\alpha}_{\mathrm{TRIG}}\right)+\epsilon_{2} \cos \left(T_{\mathrm{TRIG}}\right) \\
& \alpha_{R A T E}=5 \mathrm{deg} / \mathrm{s} \\
& \epsilon_{1}=0.01 \mathrm{~kg} / \mathrm{rad} \\
& \epsilon_{2}=0.01 \mathrm{~s} / \mathrm{m}
\end{aligned}
$$

Due to the error controls being adjoined to the mass EOM, an error will result in the vehicle propellant. This will either add or remove propellant from the final problem. It is therefore necessary to reduce both $\epsilon$ 's until the error is insignificant. Additionally each individual term of Eq. (5.3b) appears to have different dimensions. However, another variable, $\mathrm{k}$, is multiplied into each error term. This variable is dimensionally consistent with its control. When solving the problem, only the product of $\epsilon$ and $\mathrm{k}$ is considered and therefore one of them can be assumed to be one. Eq. (5.4a) describes the situation. 


$$
\begin{aligned}
& \dot{m}=-\frac{T}{g_{0} I s p}+\epsilon_{1} k_{1} \cos \left(\dot{\alpha}_{\mathrm{TRIG}}\right)+\epsilon_{2} k_{2} \cos \left(T_{\mathrm{TRIG}}\right) \\
& k_{1}=1 \mathrm{rad} / \mathrm{s} \\
& k_{2}=1 \mathrm{~N}
\end{aligned}
$$

The optimal control law for the angle of attack rate, $\dot{\alpha}$, becomes very simple as compared to the previous scenario and is shown in Eq. (5.5). Similarly, the optimal control law for thrust is shown in Eq. (5.6). Note that most aircraft do not measure angle of attack rate, this is not an issue because the history of $\alpha$ still exists from the state equation and that can be used for actual flight plans.

$$
\begin{gathered}
u_{T R I G}=\left\{\begin{array}{l}
\arctan \left(\frac{\lambda_{\dot{\alpha}_{T R I G}}}{\epsilon \lambda_{\text {mass }}}\right) \\
\arctan \left(\frac{\lambda_{\dot{\alpha}_{T R I G}}}{\epsilon \lambda_{\text {mass }}}\right)+\pi
\end{array}\right. \\
\arctan \left(\frac{T_{\text {TRIX }}\left(\frac{\lambda_{v} \cos \alpha}{m}+\frac{\lambda_{\gamma} \sin \alpha}{m v}-\frac{\lambda_{m}}{g_{0} I_{S P}}\right)}{2 \epsilon \lambda_{\text {mass }}}\right) \\
\arctan \left(\frac{T_{M A X}\left(\frac{\lambda_{v} \cos \alpha}{m}+\frac{\lambda_{\gamma} \sin \alpha}{m v}-\frac{\lambda_{m}}{g_{0} I_{S P}}\right)}{2 \epsilon \lambda_{\text {mass }}}\right)+\pi
\end{gathered}
$$

\subsection{Example Problem}

A simplified analysis was conducted to help prove the theory that $\dot{\alpha}$ as a control overcame the transcendental problem and still found an optimal solution and that the epsilon-Trig Regularization method would solve the bang-bang controls. This opportunity was also used to begin solving powered flight optimal trajectory problems. 
Therefore a rocket powered missile trajectory was studied and solved. The problem statement is the same Eqs. (4.3) with the modified equation of motion in Eq. (5.3b). The objective is to minimize the time taken to strike a target at a predetermined location.

Some of the performance parameters of the rocket can be found in Table 5.1. The thrust expression is a product of the throttle and maximum thrust capacity of the rocket, resulting in a thrust output that varies between 0 and that maximum possible thrust, $T_{M A X}$. The rocket characteristics seen in both Table 5.1 and 5.2 were closely based on the Lockheed Martin ATACMS 140 1A solid rocket [3].

Table 5.1. Constants for the rocket missile problem.

\begin{tabular}{lc} 
Parameter & Value \\
\hline $\mathrm{T}_{M A X}(\mathrm{kN})$ & 80 \\
\hline$I_{s p}(\mathrm{~s})$ & 400 \\
\hline
\end{tabular}

The boundary conditions for the problem are shown in Table 5.2. These conditions are chosen to be a similar problem as the scramjet powered problem based on the JTOH roadmap. Despite some differences the two problems will be compared to one another.

The state histories are shown in Fig. 5.1 and the control histories are shown in Fig. 5.2. A comparison is made between the results obtained by OCT using the Matlab based solver, BVP4c, and the results obtained using current state of the art optimal control solver, GPOPS-II. The minimum time to strike a predetermined target for such a rocket powered missile is found to be $761 \mathrm{~s}$. The results are in excellent agreement and show that high quality solutions that satisfy necessary conditions of optimality can be obtained using OCT with the newly proposed $\dot{\alpha}$ control.

The missile in this problem does not rely on the atmosphere to generate thrust because the rocket contains both oxygen and fuel. As a result, the vehicle in an optimal trajectory will want to climb out of the thicker portions of the atmosphere 
Table 5.2. Initial and final conditions for rocket missile problem.

\begin{tabular}{lcc} 
Attribute & Initial Value & Final Value \\
\hline Time $(\mathrm{s})$ & 0 & free \\
\hline Altitude $(\mathrm{km})$ & 18 & 0 \\
\hline Downrange $(\mathrm{km})$ & 0 & 1700 \\
\hline Velocity $(\mathrm{m} / \mathrm{s})$ & 1100 & free \\
\hline$\gamma\left({ }^{\circ}\right)$ & free & free \\
\hline Mass $(\mathrm{kg})$ & 3600 & 2000 \\
\hline$\alpha\left(^{\circ}\right)$ & free & free \\
\hline
\end{tabular}

and travel downrange at large altitudes. This climb can be seen in the energy plot. The vehicle travels the entire downrange at altitude, waiting until directly above the target to dive into the thick portions of the atmosphere. This architecture minimizes the total drag force experienced over the entire trajectory.

From the mass history plot it is seen that the rocket expends the entire propellant mass at the beginning of the trajectory as well as doing so at the maximum possible rate. These are general features of optimality. This is in agreement with the thrust history plot.

Once all of the fuel is burned, the powered hypersonic missile becomes a glide hypersonic missile. A common feature of glide hypersonic vehicles is oscillation [40]. The altitude-downrange plot shows a few oscillations occurring. The trajectory contains many known features of optimality.

The large spike in the angle of attack rate plot is a numerical artifact. It is a consequence of the inaccuracies of GPOPS. The OCT also contains the spike, but it is a result of the choice of adjoining EOM for the error control. A solution to this problem is offered in Section 6.3.1. 

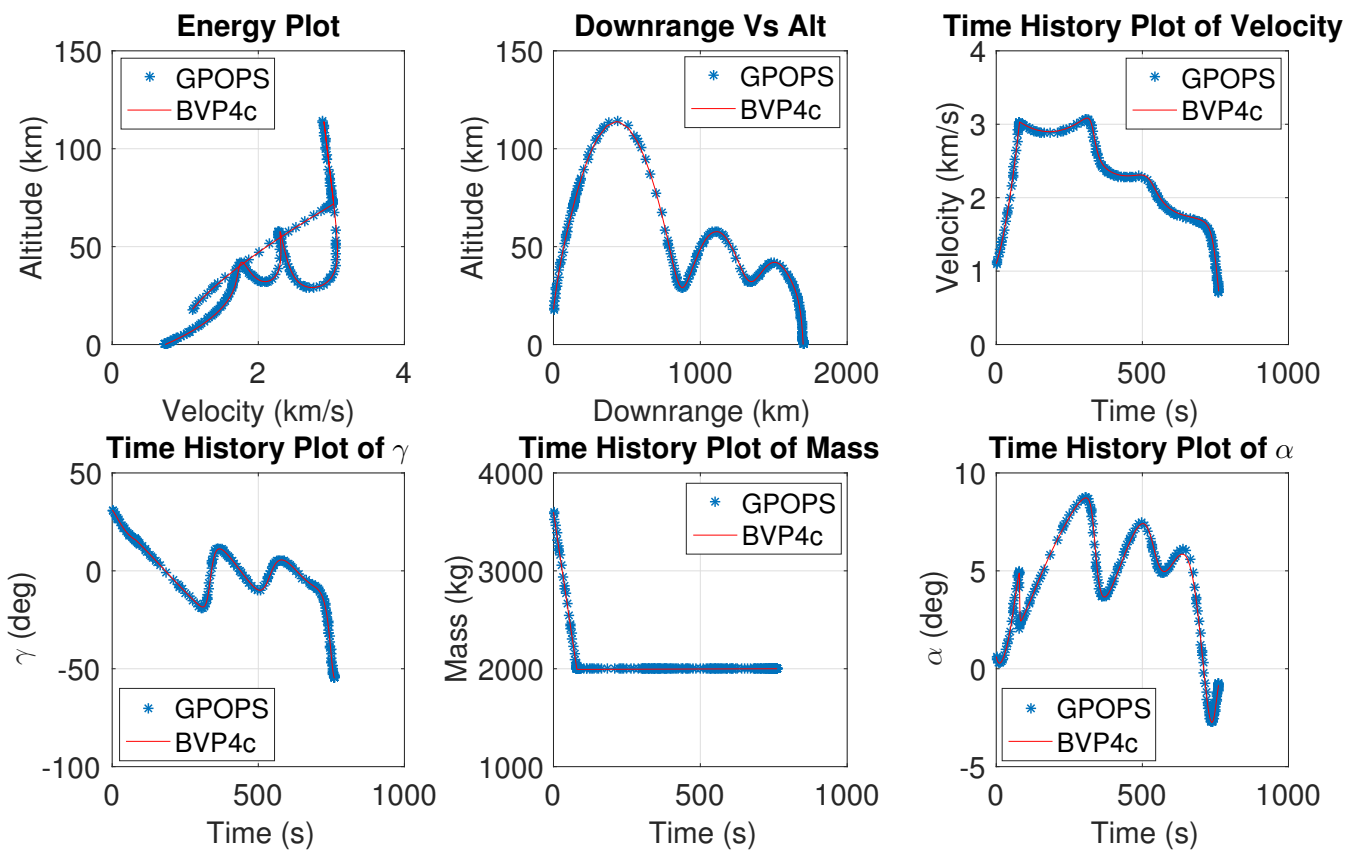

Figure 5.1. State histories using bvp4c and GPOPS-II.
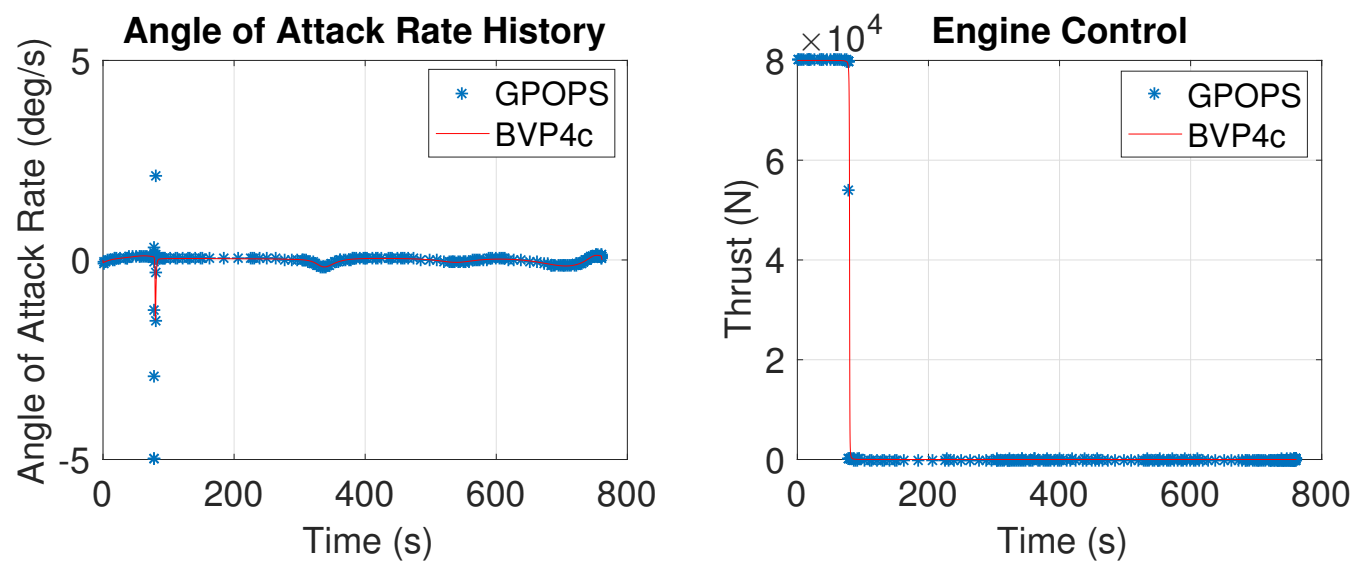

Figure 5.2. Control histories using bvp4c and GPOPS-II. 
Despite reducing both error parameters to low magnitudes, their effect is still present in the problem. By integrating the mass EOM over the entire trajectory, the cumulative propellant burned is $1608 \mathrm{~kg}$. This is a half percent error in available fuel and the added mass is a violation of the real physics involved. The error, as required by the regularization approach, is insignificant.

Angle of attack rate history shows a small discrepancy between the two solvers. The saturation of the control in GPOPS's solution is an example in the difference in solution qualities. If the control did not have the lower bound of -5 , the downward singularity would have continued, affecting the solution even more.

Table 5.3 provides information on the objective function value and the solution time of both solution methods. As is expected based on the agreement in the state and control figures, the objective functions are within a small tolerance of each other. GPOPS is able to solve this simple problem, relative to hypersonic trajectories, rapidly while OCT requires more time.

Table 5.3. Solver Performance.

\begin{tabular}{lcc} 
Solver & Objective Function Value (s) & Solution Time (s) \\
\hline GPOPS & 761.4 & 11.2 \\
\hline OCT & 761.4 & 247 \\
\hline
\end{tabular}




\section{THE SCRAMJET PROBLEM}

\subsection{The Thrust Model}

As previously discussed, trajectory optimization of air-breathing vehicles has traditionally been conducted through the use of tabulated engine models. This work introduces an analytic approach to modeling the scramjet propulsion system within the optimization framework allowing OCT to leverage mathematics to obtain higher quality solutions for complicated problems. The analytic relationships may also offer deeper insight into better performing operating ranges for scramjets.

\section{Analytic Model}

The scramjet model used in this paper is a parametric ideal scramjet cycle analysis developed in Ref. 12. This cycle analysis is fundamentally based on the Brayton cycle. The major assumptions include: an isentropic inlet, a constant pressure combustion process, an isentropic nozzle, and a constant pressure heat rejection process. The flow exiting the nozzle measures a static pressure that is equal to the freestream ambient static pressure.

The following process relates the freestream conditions of Mach number and temperature to the scramjet engine operation (specific thrust and fuel-air ratio) to model the coupled performance. $\tau_{r}$ is the inlet temperature ratio and Eq. (6.1) is the result of an adiabatic inlet.

$$
\tau_{r}=1+\frac{\gamma-1}{2} M_{0}^{2}
$$

$T_{M a x}^{\prime}$ is the total temperature at the nozzle exit and can be found through Eq. (6.2), where $T_{M a x}$ is the temperature limit of the combustor material. In a scramjet engine, combustion occurs at supersonic speeds, this has the benefit that the engine walls 
never experience the total temperature present in the burner and therefore eases the cooling requirements.

$$
T_{\text {Max }}^{\prime}=T_{\text {Max }}\left(1+\frac{\gamma_{r}-1}{2} M_{c}^{2}\right)
$$

$\tau_{\lambda}$ is created for a more compact evaluation of the specific thrust and fuel-air ratio.

$$
\tau_{\lambda}=\frac{T_{M a x}^{\prime}}{T_{0}}
$$

The ratio of mass flow rate of fuel injected into the burner to the mass flow rate of air passing through the engine, f, has been used as a parameter for throttling the engine directly. However, in this work, $\dot{m}_{0}$ will be throttled through a variable geometry inlet. Within the cycle analysis, $\mathrm{f}$ is needed to calculate specific impulse and can be found by applying the steady-flow energy equation to the control volume across the combustion chamber. In other words, using the first law of thermodynamics:

$$
f=\frac{c_{p} T_{0}}{h_{p r}}\left(\tau_{\lambda}-\tau_{r}\right)
$$

The other performance variable of the cycle analysis is specific thrust. Both specific thrust and fuel-air ratio are parameters scalable by the engine and more specifically $\dot{m}_{0}$. They are an indication of engine efficiency. A smaller $f$ would result in less fuel burned for a given mass flow rate, however the larger the specific thrust, the more thrust that is produced for that same mass flow rate.

$$
\begin{gathered}
\frac{F}{\dot{m}_{0}}=\frac{V_{0}}{g_{c}}\left(\sqrt{\frac{\tau_{\lambda}}{\tau_{r}}}-1\right) \\
\dot{m}_{0}=\rho A_{c} V_{0}
\end{gathered}
$$

Figure 6.1 shows the performance of the scramjet engine across a range of freestream Mach numbers at $26 \mathrm{~km}$ altitude. In actual flight, the scramjet will not operate below a flight Mach number of approximately 4. Finally, the end results of the ideal cycle analysis are given in Eqs. (6.7) and (6.8). These are the values that feed back into the equations of motions in Eq. (4.3) and complete the coupled nature of the flight dynamics and propulsion system. 

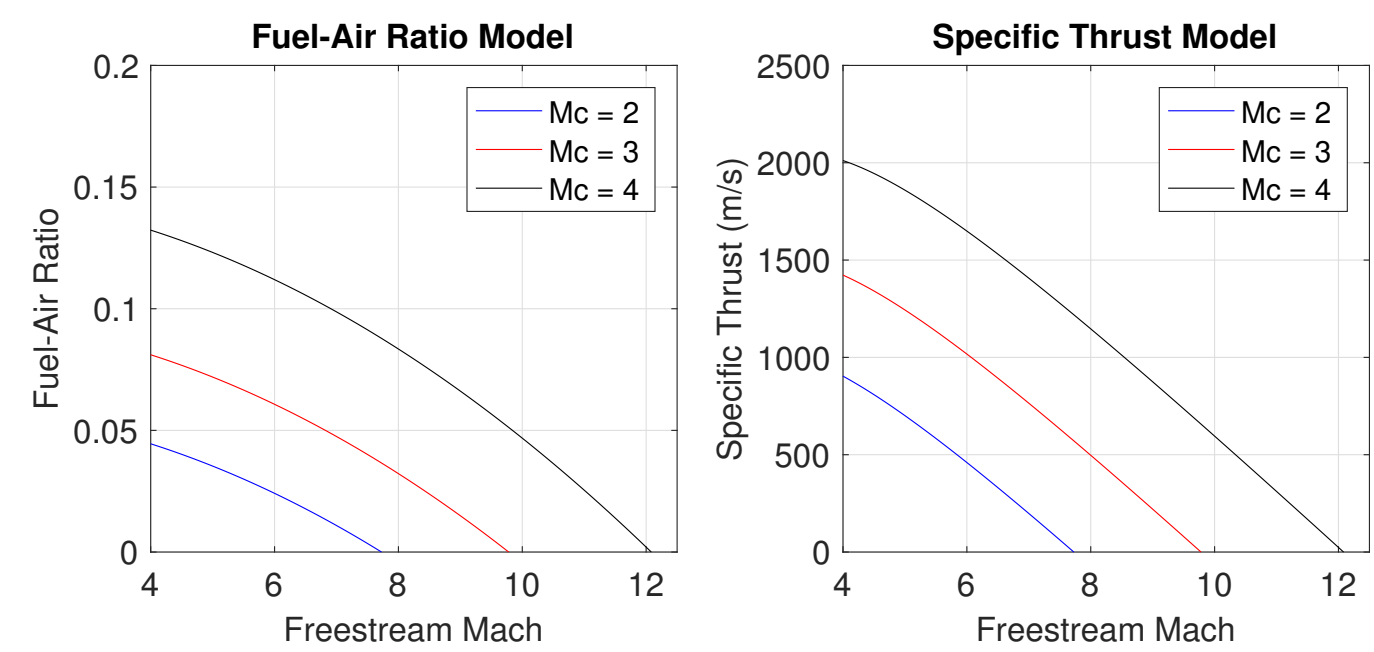

Figure 6.1. Range of scramjet engine model performance at $26 \mathrm{~km}$ altitude.

$$
\begin{gathered}
T=\frac{F}{\dot{m}_{0}} \dot{m}_{0} \\
I_{s p}=\frac{\frac{F}{\dot{m}_{0}}}{g_{0} f}
\end{gathered}
$$

\subsection{Scramjet Engine Bounding}

As seen in Fig. 6.2, the unbounded thrust model could be used for flight Mach numbers outside of the operating range of the scramjet. The saturation function arctan was used to enforce only valid operating conditions. Fig. 6.2 shows the behavior of the thrust model with the saturation functions applied.

To manipulate the thrust model, $\tau_{\lambda}$ was inserted into the arctan saturation function seen in Eq. (6.9). The lower $\left(q_{1}\right)$ and upper $\left(q_{2}\right)$ bounds of the saturation function are the dynamic pressure operational limits of the scramjet and are where the switching occurs. In this work, the scramjet was assumed to produce thrust in dynamic pressures greater than the vehicle would operate. Therefore $q_{2}$ was set to a sufficiently large value. How quickly the saturation function switches is controlled by $q_{\text {scale. }}$. There 
is potential to use this for a transient phase between starting and unstarting the engine. For this work $q_{\text {scale }}$ was set so that switching would occur over a short period of time. While within the desired bounds of dynamic pressure, $\tau_{\lambda}^{*}$ equals $\tau_{\lambda}$, otherwise it becomes equal to $\tau_{r}$. This results in Eq. (6.4) and Eq. (6.5) producing a value of zero, meaning no propellant is burned, nor thrust achieved.

$$
\tau_{\lambda}^{*}=\tau_{r}+\left(\tau_{\lambda}-\tau_{r}\right) \frac{\left[\arctan \left(q_{\text {scale }}(q-q 1)\right)-\arctan \left(q_{\text {scale }}(q-q 2)\right)\right]}{\pi}
$$

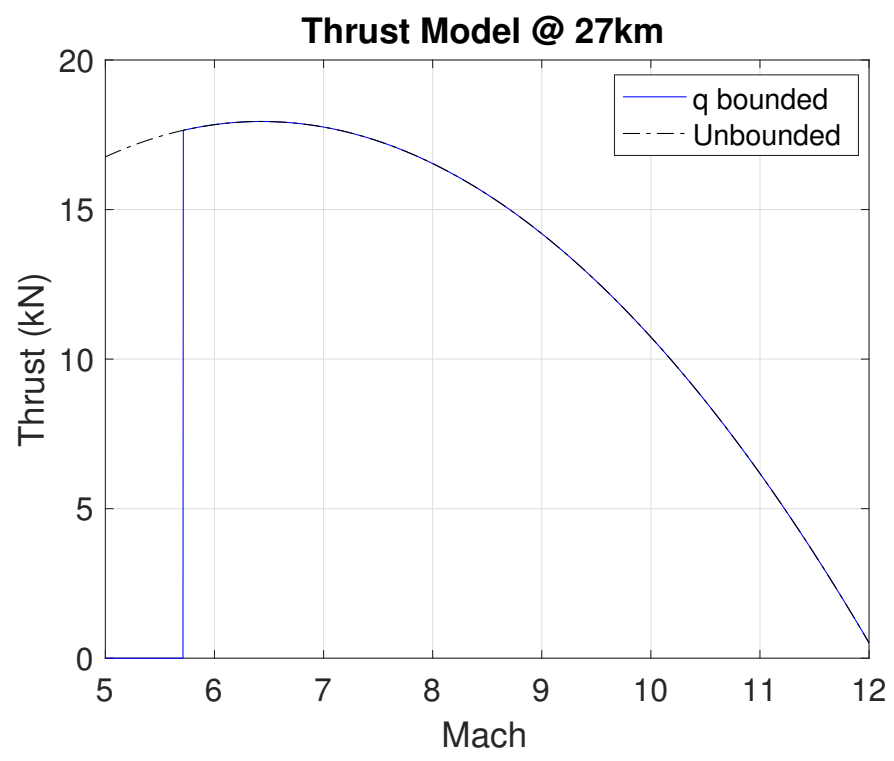

Figure 6.2. Scramjet engine operation limited by dynamic pressure.

The engine model can be bounded by Mach number with the same approach. This may be necessary for future air-breathing hypersonic work. In the solutions presented below, the Mach number remained in a valid range and therefore no Mach bounding was conducted.

\subsection{Scramjet Solutions}

The prompt, regional strike problem statement from Chapter 4 was solved . However, it was now solved using the engine model described above as opposed to using 
the rocket booster discussed in Chapter 5. The problem statement is expressed again in Eqs. 6.10 because of key changes. The thrust control was switched from a generic thrust concept to the engine inlet area control, $A$, simulating a variable geometry inlet by way of adjusting wedge angles. The inlet was assumed to be in the optimal position during the entire trajectory. Future work can improve the modeling of the variable geometry inlet and determine if the performance benefits are worth the expense. However, this was a convenient control for the problem because once simplified, the only equation relevant to the EOMs that used inlet area was thrust. Additionally, the inlet area is linear in the thrust equation, resulting in the same ban-bang nature as before, see Eq. 6.10h.

Other changes include the EOM for mass being simplified to $-\dot{m}_{f} f$. This was to simplify the control law which converted the inlet area control into a bang-bang control. A benefit found is that the optimal solution can be more easily verified through engineering judgment. The error controls are still adjoined to the mass EOM, but the units and magnitude of the error parameters, $\epsilon_{1}$ and $\epsilon_{2}$ have changed.

Minimize:

$$
J=t_{f}
$$

Subject to:

$$
\begin{aligned}
\dot{h} & =v \sin \gamma \\
\dot{\theta} & =\frac{v \cos \gamma}{r} \\
\dot{v} & =\frac{T \cos \alpha-D}{m}-\frac{\mu \sin \gamma}{r^{2}} \\
\dot{\gamma} & =\frac{(L+T \sin \alpha)}{m v}+\left(\frac{v}{r}-\frac{\mu}{v r^{2}}\right) \cos \gamma \\
\dot{m} & =-\dot{m}_{0} f+\epsilon_{1} \cos \left(\dot{\alpha}_{T R I G}\right)+\epsilon_{2} \cos \left(A_{T R I G}\right) \\
\dot{\alpha} & =\alpha_{\text {rate }} \sin \left(\dot{\alpha}_{T R I G}\right)
\end{aligned}
$$


Where:

$$
\begin{aligned}
& A=A_{M A X}\left(\frac{1+\sin A_{T R I G}}{2}\right) \\
& u_{1}=\dot{\alpha}_{T R I G} \\
& u_{2}=A_{T R I G} \\
& \epsilon_{1}=0.5 \mathrm{~kg} / \mathrm{rad} \\
& \epsilon_{2}=1 e-13 \mathrm{~kg} /\left(\mathrm{m}^{2} \mathrm{~s}^{1}\right)
\end{aligned}
$$

The expression for thrust in this problem contained more variables due to the coupling of the propulsion system with the atmosphere. From the necessary conditions of optimality, the new control law is significantly longer than Eq. (5.6). Eqs. (6.14) and (6.15) show the computed analytic control laws for this problem. The rapidly increasing complexity of the control law can be seen in $A_{T R I G}$.

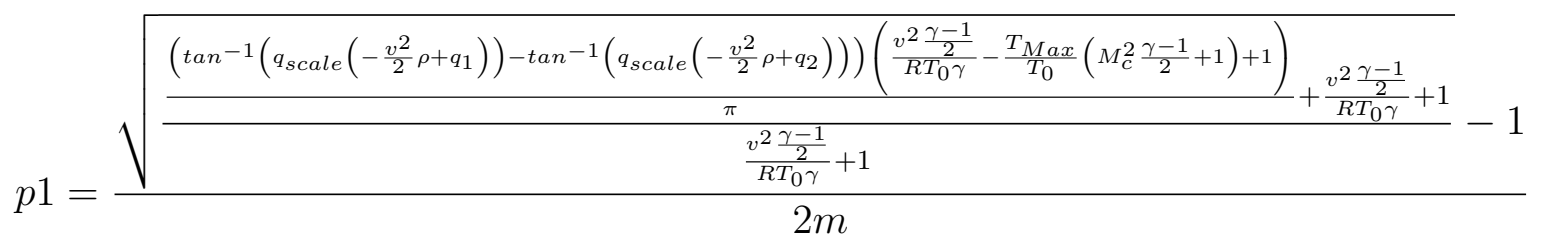

$p 2=\frac{\left(\tan ^{-1}\left(q_{\text {scale }}\left(-\frac{v^{2}}{2} \rho+q_{1}\right)\right)-\tan ^{-1}\left(q_{\text {scale }}\left(-\frac{v^{2}}{2} \rho+q_{2}\right)\right)\right)\left(\frac{v^{2} \frac{\gamma-1}{2}}{R T_{0} \gamma}-\frac{T_{M a x}}{T_{0}}\left(M_{c}^{2} \frac{\gamma-1}{2}+1\right)+1\right)}{2 \pi h_{p r}}$

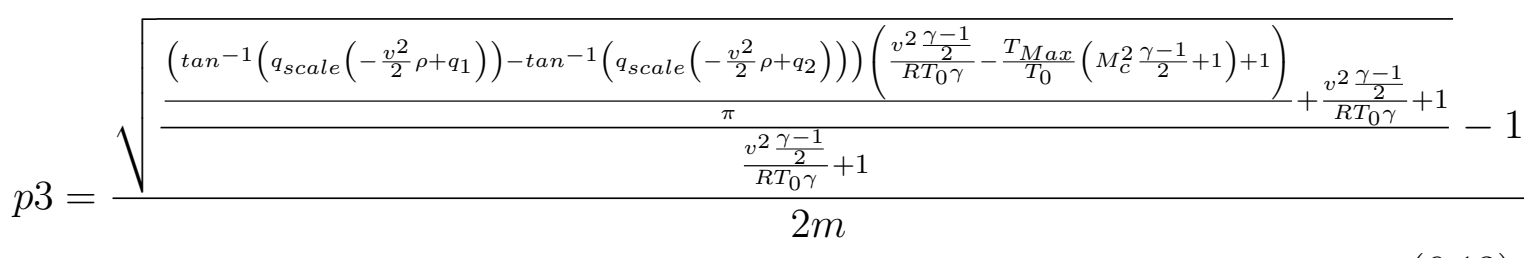




$$
\begin{aligned}
& A_{T R I G}=\left\{\begin{array}{l}
\arctan \left(\frac{\left(A_{M a x} \rho_{0} v e^{-h / H}\left(\lambda_{v} v^{2} \cos (\alpha) p 1-\lambda_{\text {mass }} T_{0} C_{p} p 2+\lambda_{\text {gam }} \sin (\alpha) p 3\right)\right)}{\epsilon_{2} \lambda_{\text {mass }}}\right) \\
\arctan \left(\frac{\left(A_{M a x} \rho_{0} v e^{-h / H}\left(\lambda_{v} v^{2} \cos (\alpha) p 1-\lambda_{\text {mass }} T_{0} C_{p} p 2+\lambda_{\text {gam }} \sin (\alpha) p 3\right)\right)}{\epsilon_{2} \lambda_{\text {mass }}}\right)+\pi
\end{array}\right. \\
& \dot{\alpha}_{\text {TRIG }}=\left\{\begin{array}{l}
\arctan \left(\frac{\alpha_{\text {rate }} \lambda_{\alpha}}{\epsilon_{1} \lambda_{\text {mass }}}\right) \\
\arctan \left(\frac{\alpha_{\text {rate }} \lambda_{\alpha}}{\epsilon_{1} \lambda_{\text {mass }}}\right)+\pi
\end{array}\right.
\end{aligned}
$$

An expanded list of variables are required for the thrust calculation. These variables describe both necessary atmospheric and engine parameters and are seen in Table 6.1. These are considered the baseline values for the parameters in this study and a few will be varied later in Chapter 7. Many of the parameters are ideal values for a perfect gas or simplified atmosphere. This includes the specific heat ratio, $\gamma$, the Earth's atmospheric gas constant, $R$, and the heat capacity at constant pressure, $c_{p}$. $T_{0}$, the freestream temperature, is a constant value due to the exponential atmosphere assumption. The fuel heating value corresponds to the use of JP-7. This fuel, used in the SR-71, is a popular choice because of its endothermic properties allowing for active cooling while cracking the fuel before combustion. The selected lower dynamic pressure limit $\left(q_{1}\right)$ for sustainable engine operation is from [37]. $q_{2}$ is defined so that the vehicle will not encounter the limit. Future work should address lowering it to appropriate values.

Table 6.2 provides the boundary conditions placed on the scramjet problem. The majority of the initial conditions are recognized from the previous rocket problem and are set to values that are obtainable by a booster and conducive to scramjet operation. The terminal boundary conditions provide both the target location and the dry weight of the missile. 
Table 6.1. Values for scramjet engine input.

\begin{tabular}{lc} 
Parameter & Value \\
\hline$\gamma(\mathrm{nd})$ & 1.4 \\
\hline $\mathrm{R}\left(\mathrm{m}^{2} / \mathrm{s}^{2} \mathrm{~K}\right)$ & 287.058 \\
\hline $\mathrm{c}_{\mathrm{p}}\left(\mathrm{m}^{2} / \mathrm{s}^{2} \mathrm{~K}\right)$ & 1004 \\
\hline $\mathrm{h}_{\mathrm{pr}}\left(\mathrm{m}^{2} / \mathrm{s}^{2}\right)$ & 43903250 \\
\hline $\mathrm{M}_{\mathrm{c}}(\mathrm{nd})$ & 3 \\
\hline $\mathrm{T}_{\mathrm{Max}}(\mathrm{K})$ & 1600 \\
\hline $\mathrm{T}_{0}(\mathrm{~K})$ & 230 \\
\hline $\mathrm{A}_{\max }\left(\mathrm{m}^{2}\right)$ & 0.3 \\
\hline $\mathrm{q}_{1}\left(\mathrm{~kg} / \mathrm{ms}^{2}\right)$ & 20000 \\
\hline $\mathrm{q}_{2}\left(\mathrm{~kg} / \mathrm{ms}^{2}\right)$ & 3500000 \\
\hline
\end{tabular}

Table 6.2. Initial and final conditions for scramjet missile problem.

\begin{tabular}{lcc} 
Attribute & Initial Value & Final Value \\
\hline Time (s) & 0 & free \\
\hline Altitude (km) & 20 & 0 \\
\hline Downrange (km) & 0 & 1700 \\
\hline Velocity (m/s) & 1300 & free \\
\hline Flight Path Angle & free & free \\
$\left(^{\circ}\right)$ & & \\
\hline Mass (kg) & 1300 & 600 \\
\hline Angle of Attack $\left(^{\circ}\right)$ & free & free \\
\hline
\end{tabular}

Observing the solutions in Figs. 6.3, a different trajectory architecture from the rocket solution is found. After beginning from the boosted state, the missile immedi- 
ately dives to a thicker portion of the atmosphere. This solution leads to the vehicle experiencing larger dynamic pressures which has a proportional affect on the thrust generated. The dive also corresponds to increased drag force from the atmosphere, but the vehicle still accelerates rapidly as seen by the steep rise in the velocity history plot. Quickly regaining altitude after the dive, the result of the altitude deviation is a missile traveling over a one $\frac{\mathrm{km}}{\mathrm{s}}$ faster by the time it enters a cruise phase.

The mass history plot illustrates how the vehicle burns through its fuel the most at the beginning and end of the trajectory. The connection between these two phases is that they both occur lower in the atmosphere where oxygen is available in larger quantities. The first substantial fuel burn is optimal since it allows the vehicle to accelerate rapidly. The second substantial fuel burn is used to offset some of the significant deceleration during the descent phase. The last, two kilometers of decent are met with a $20 \%$ reduction in velocity. This is also the reason that the vehicle nearly reaches the terminal downrange at altitude and then performs a steep dive to the target. These are features expected in the optimal solution.

A lapse in the model exists with the captured inlet air flow. In flight, when the angle of attack becomes too large, oblique shocks at the engine inlet lead to flow spill making it difficult for the engine to consume enough air for combustion. This feature is not captured in the model, but an assumption is made that the variable geometry inlet adjusts accordingly. However in this particular trajectory the angle of attack remains small and the Mach number constant over nearly the entire history of the trajectory. Therefore the issue of spilled flow is significantly reduced and a strong argument can be made that only small penalties would be incurred with a fixed inlet. Different trajectories may result rely more on the variable geometry inlet. Studies can be conducted that consider the trade-off between the large expense of a variable inlet and the performance penalties of a fixed inlet.

Despite the engine bounding feature in the indirect solution (BVP4c), the direct solver solution (GPOPS) agrees closely. The dynamic pressure of the trajectory did not lower enough to activate the $q_{1}$ bound. One main reason is that the vehicle would 
have to reduce velocity or climb to altitudes without enough oxygen for sustainable combustion. Both options would increase the time to target and therefore were not optimal.
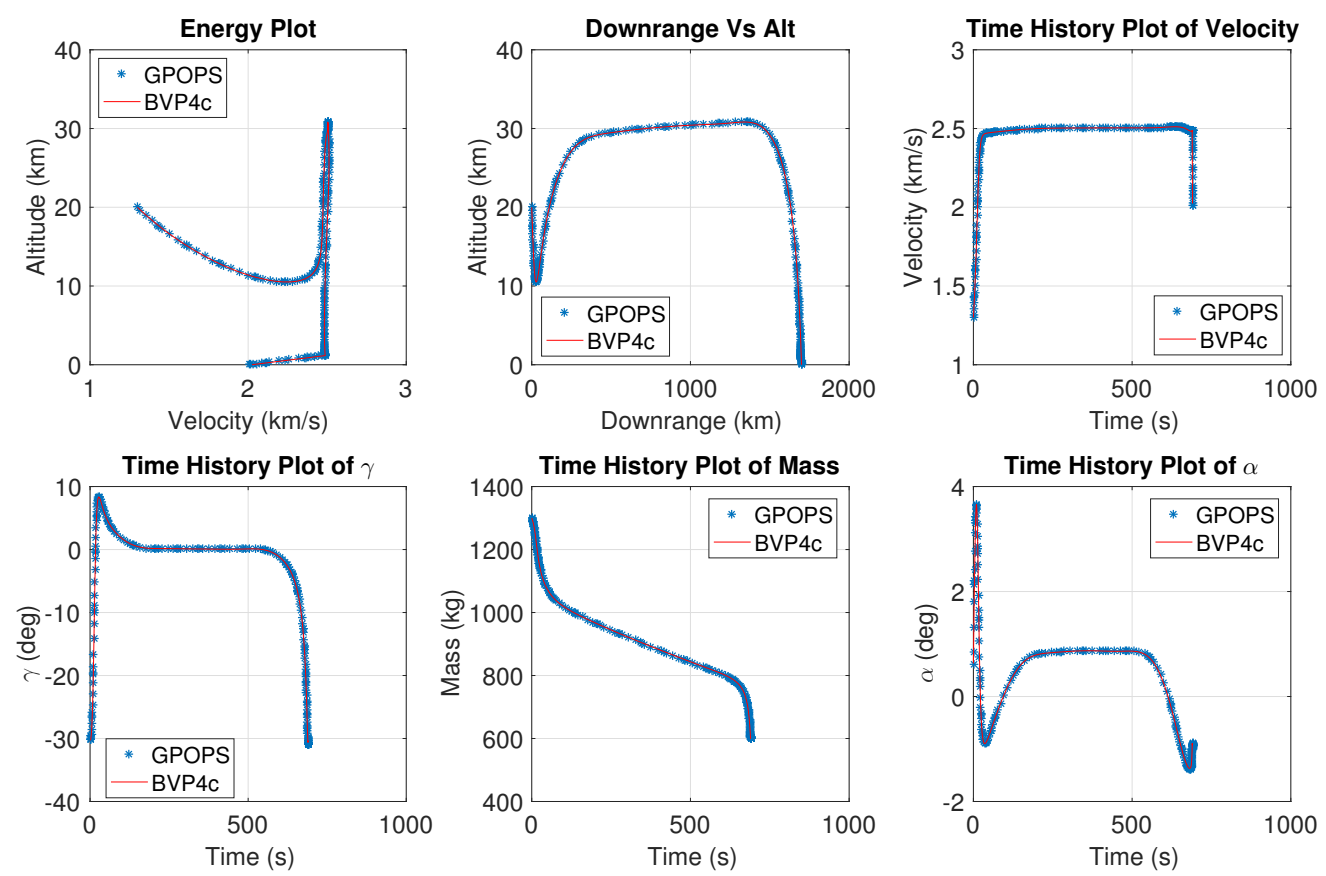

Figure 6.3. State histories using GPOPS-II and BVP4C.

In Fig. 6.4 the solution to the optimal controls are shown. The engine control history shows that the vehicle burns as much fuel as it can for the mass flow rate of air that passes into its inlet. It does this until all the fuel reserves are depleted. This structure is in agreement with previous works and is optimal [5].

In Fig. 6.5, the Mach number range is seen to be neither too low (unsustainable operation) or too high (negative thrust from the analytic model) to warrant Mach bounding. The dynamic pressure over the course of the trajectory peaks twice, to values much higher than any modern vehicle can withstand. This phenomenon has been seen in other optimal problems as well [14]. Because these dynamic pressures 

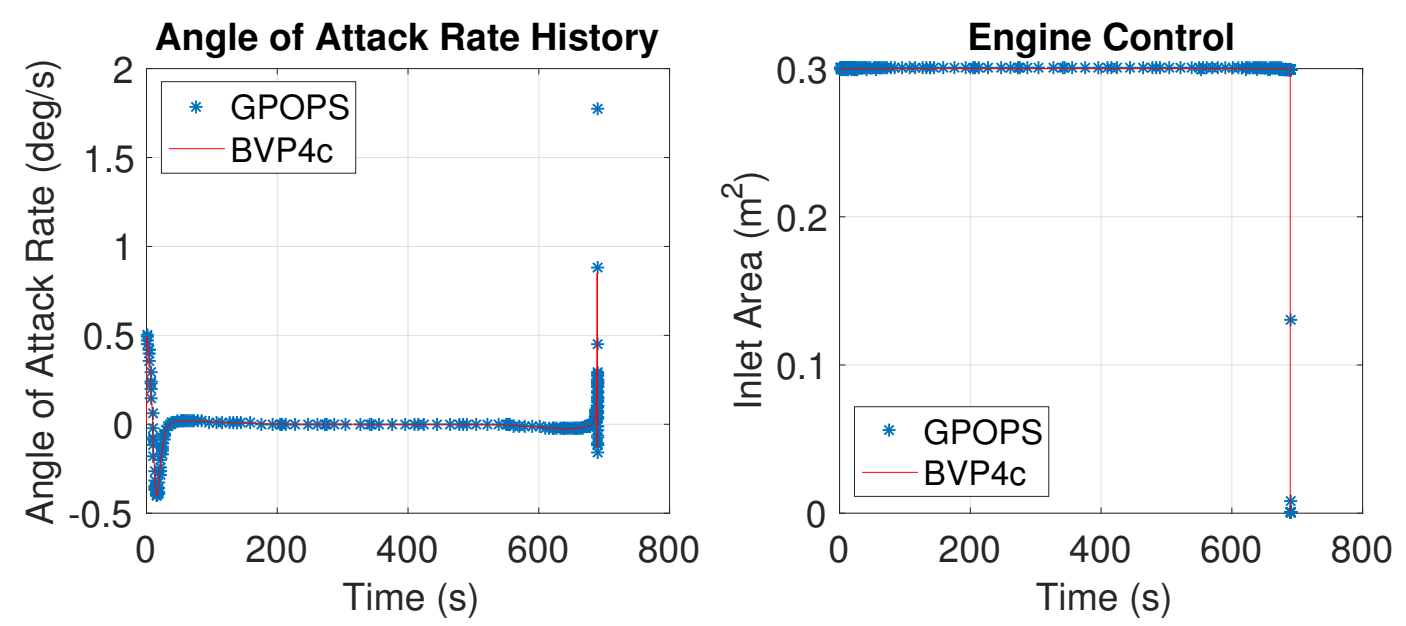

Figure 6.4. Control histories using GPOPS-II and BVP4C.

cannot be flown in reality, the solutions presented are unrealistic, but represent the capabilities of this engine-trajectory bridging.
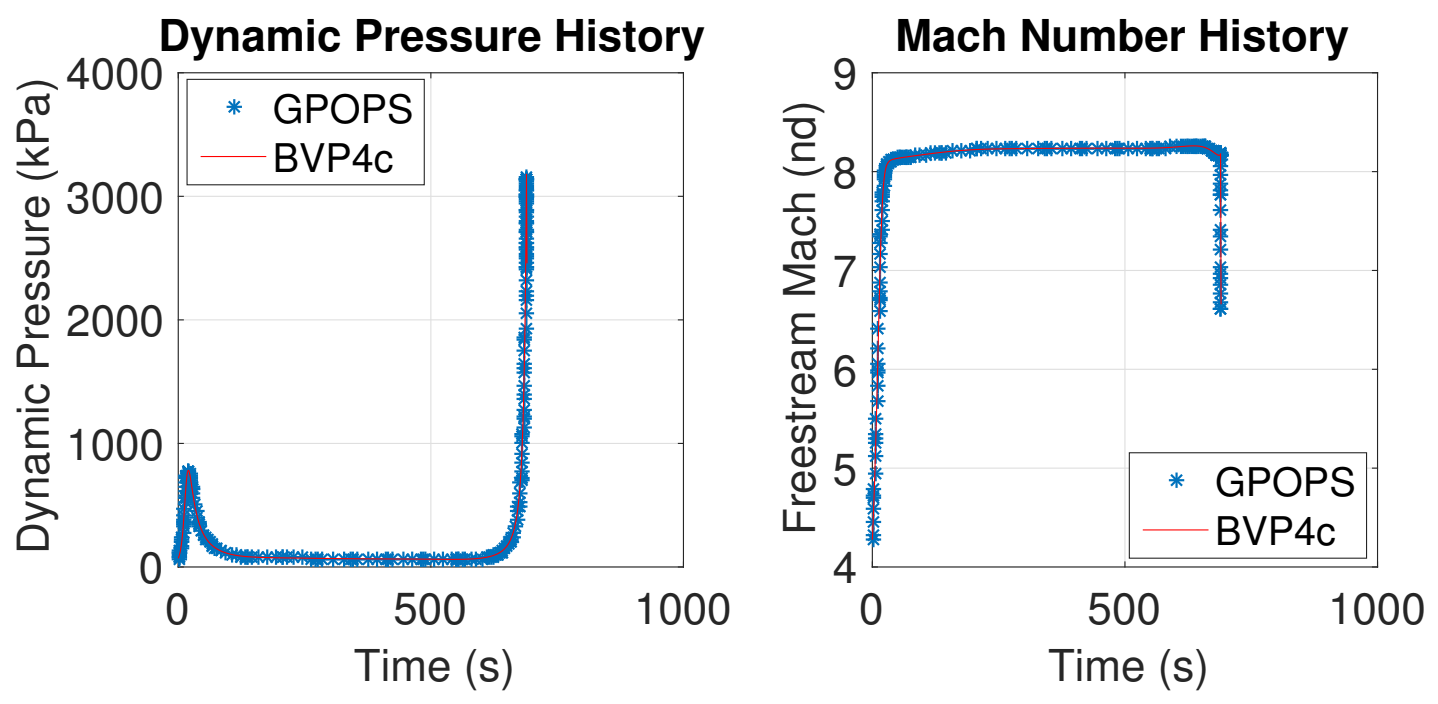

Figure 6.5. Dynamic pressure and Mach histories over the trajectory.

A benefit of solving different vehicle options quickly, in the scope of the overall mission is that designs can be compared rapidly and at the start of the schedule. 
For example, the missile from Chapter 5 powered by a rocket can now be compared with the scramjet powered missile solution solved above. The objective minimizes time to target, and, from that objective alone, the missile powered by the scramjet is the better design, reaching the target about a minute before the rocket. However, knowing the performance of both vehicles can allow other trade-offs to be considered. The rocket has an initial mass that is nearly three times larger than the scramjet. The size discrepancy is a result of the rocket needing to carry both the oxygen and fuel whereas the scramjet will receive its oxygen from the atmosphere. Integrating the rocket into a missile is significantly cheaper than a scramjet. The scramjet needs to be accelerated to a larger Mach number before it can be started and therefore its trajectory begins at a higher altitude and larger velocity than the rocket. This mission has more phases and therefore is more complicated and risky. But the rocket climbs to much higher altitudes to reach its target. This could have implications with treaties regarding intercontinental ballistic missiles. A project manager is able to consider these trade-offs early in the design schedule and make more educated decisions on how to proceed. With a selected design, the same project leaders can set design specific requirements for a development team.

\subsubsection{Epsilon-Trig Adjoining}

Original solutions of the scramjet problem used the Epsilon-Trig regularization technique by adjoining the error control to the altitude equation of motion. A result of this decision is that the control law has the altitude costate in the denominator. The optimal solution to the minimum time problem gives the altitude costate history seen in Fig. 6.6. The zero crossings result in singularities seen in the $\dot{\alpha}$ control history in Fig. 6.7. Additionally, the singularities create a need for more nodes, and the increased number of nodes drastically increase the solution time as shown in Table 6.3. 
To resolve the singularity problem, the error control terms were moved to the mass equation of motion. Similar to the altitude EOM, the mass EOM was a good candidate because of its bounded nature. States that are unbounded either at their initial or terminal point are guaranteed to have a costate of zero at that point. Unlike, $\lambda_{h}$ in this minimum time problem, $\lambda_{\text {mass }}$ always remained negative as seen in Fig. 6.6. The singularities are eliminated, and the result is an optimal control. A significant computational speedup of nearly six times is observed.
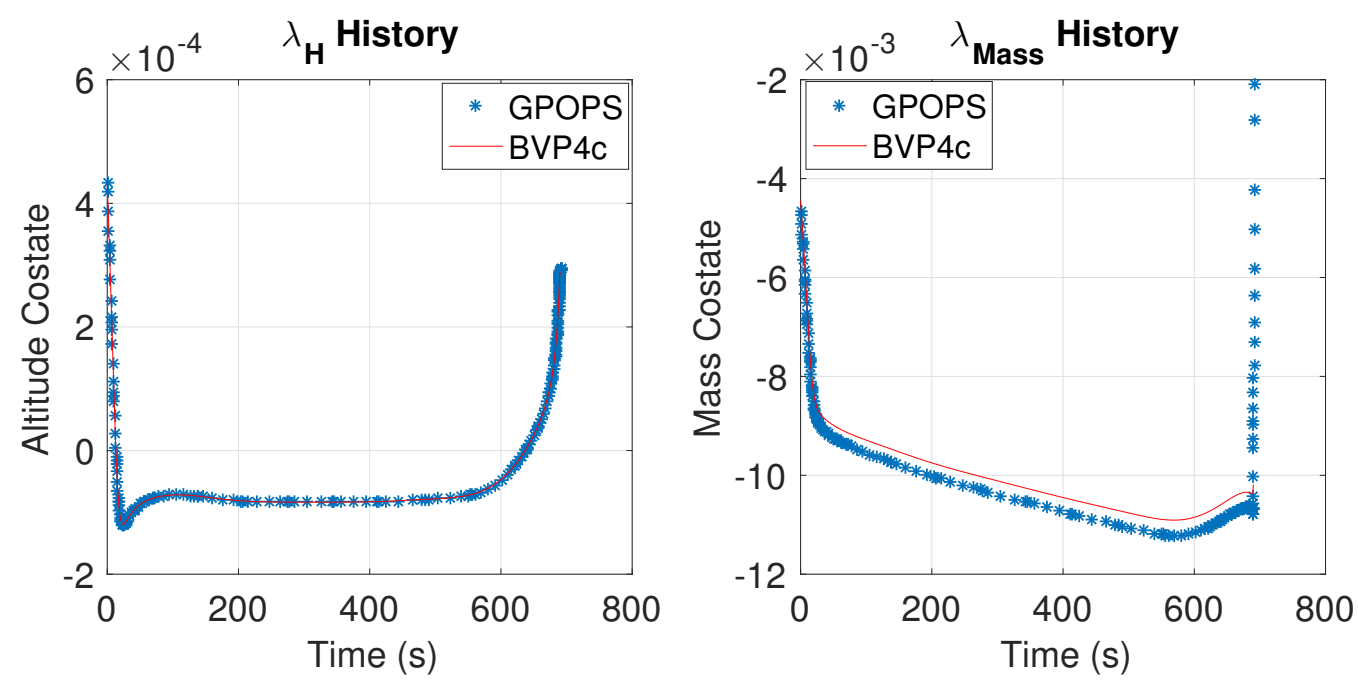

Figure 6.6. Costate histories explaining numerical spikes in controls.

Table 6.3. Solver Performance.

\begin{tabular}{lcc} 
Solver & Objective Function Value (s) & Solution Time (s) \\
\hline OCT H Adjoining & 690.5 & 2825.6 \\
\hline OCT Mass Adjoining & 690.9 & 492 \\
\hline OCT Engine Bounding & 690.7 & 912.3 \\
\hline GPOPS & 691.2 & 9 \\
\hline
\end{tabular}



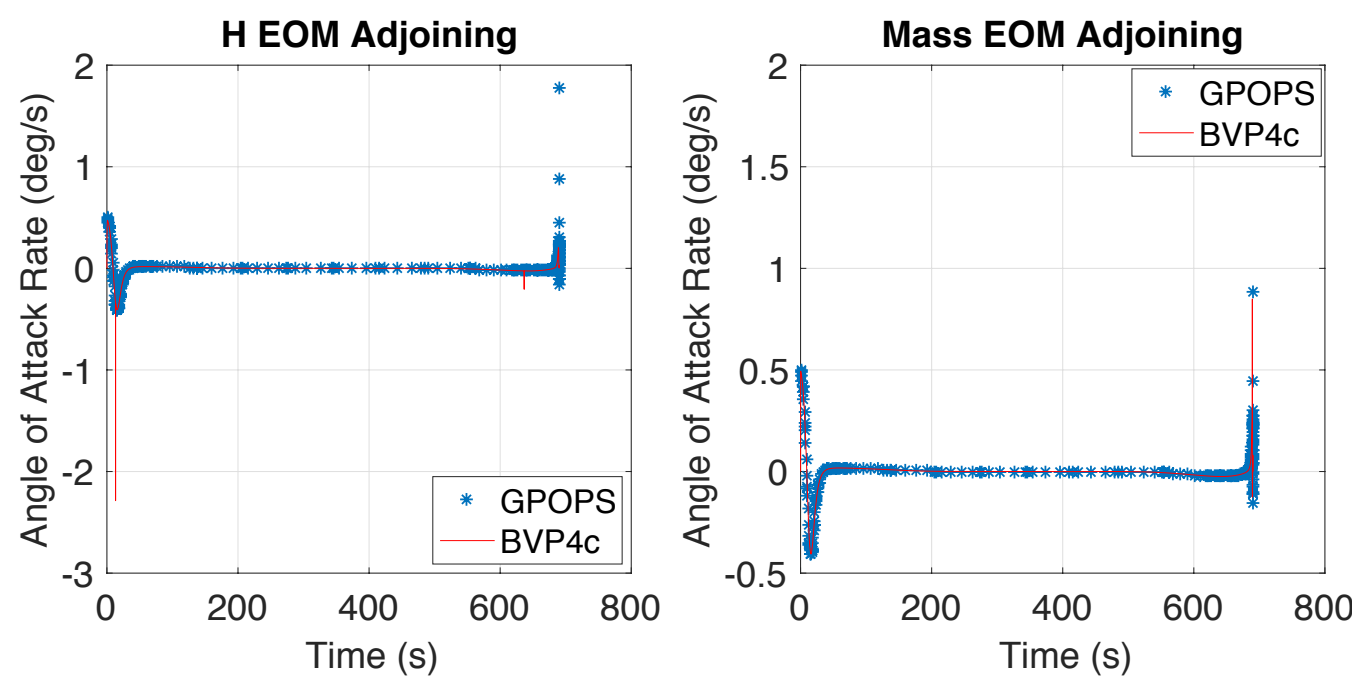

Figure 6.7. Control accuracy given adjoining methodology.

For objective functions such as minimize terminal velocity for soft landings, the terminal velocity, $V_{f}$ should approach $0 \frac{\mathrm{m}}{\mathrm{s}}$. The costate associated with velocity will be function of $V_{f}$ and therefore will also approach 0 . This state should not be used for adjoining the error control.

When the engine bounding structure was added to the indirect solver the solution time nearly doubled. This provides insight into the added complexity of the problem.

Finally, GPOPS-II was able to solve the problem within 10 seconds but it does not guarantee that necessary conditions of optimality would be satisfied. With its default settings and common techniques it could not incorporate the engine bounding. Finally, GPOPS cannot be parallelized. Current research is investigating parallelizing the indirect solver which could make its computation time competitive with GPOPS.

\subsubsection{Continuation Process}

The continuation process allows the user to start from a problem that is simple and evolve it through steps to a desired solution. To obtain the final product shown 
in this chapter, a specific continuation process was necessary to navigate the growing problem to its destination. Currently, discovering the correct process to solve the problem is a long procedure of trial and error. This process is summarized briefly:

- Step 1: Engine dynamic pressure bound $\left(q_{2}\right)$ must be started relatively low while $q_{1}$ is started at zero (non-active)

- Step 2: Terminal altitude is brought to zero

- Step 3: Terminal downrange is taken from the initial guess value to $300 \mathrm{~km}$

- Step 4: Downrange is taken slightly farther while mass is decreased

- Step 5: Terminal mass is further decreased while $q_{2}$ is increased

- Step 6: Downrange is extend while increasing $q_{2}$

- Step 7: Downrange is extended while terminal mass is decreased

- Step 8: Downrange is extend while increasing $q_{2}$

- Step 9: Downrange is extended while terminal mass is decreased

- Step 10: $\epsilon_{2}$ is increased

- Step 11: Downrange is increased to its final terminal position

- Step 12: $\epsilon_{2}$ is decreased

- Step 13: Initial altitude is brought down to initial value

- Step 14: $q_{1}$ is increased from zero to its lower, inactive bound, $20 \mathrm{kPa}$

In many problems, placing constraints on the trajectory can result in making it troublesome to obtain solutions. However, in the case of the scramjet problem, starting with a relatively low $q_{2}$ bound, confines solution space to aid the solver in finding the optimal path. Terminal states can be extended to the perimeter of this 
space. $q_{2}$ is then increased and the process repeats until all states are at the desired values. The final step of increasing the lower bound on the engine proves that the presented engine bounding technique is valid. 


\section{TRADE STUDIES}

By incorporating the engine model into the trajectory framework, it is possible to conduct trade studies on specific parameters. The solution found in Chpater 6 is used as a baseline for comparison. Maximum engine inlet area, maximum combustion temperature, combustion Mach number, and fuel were varied separately. The studies below investigate these engine parameter's effect on the overall mission.

The first parameter studied was the maximum combustion temperature. The temperature was increased by increments of $300 K$ from the baseline $1600 K$. As expected, a high combustion temperature leads to more thrust production as well as a more efficient burn. This means that the vehicle was able to reach a larger velocity, operate at a higher altitude, and ultimately hit the target sooner. These trends are seen in the figures below with the time of flight recorded in Table 7.1. The trend in the objective function shows diminishing returns as the combustion temperature continues to rise. The first $300 \mathrm{~K}$ increase in $T_{\text {Max }}$ was more beneficial then the next 300 K. From a vehicle perspective when the flight path involves larger velocities and Mach numbers the aeroheating load is significantly increased. These additional phenomena need to be considered and introduced into the modeling to gain more feedback in the consequences of certain choices.

Table 7.1. Values for scramjet engine input.

\begin{tabular}{lc} 
Tmax (K) & Time of Flight (s) \\
\hline 1600 & 691.4 \\
\hline 1900 & 632.7 \\
\hline 2200 & 587.2 \\
\hline
\end{tabular}




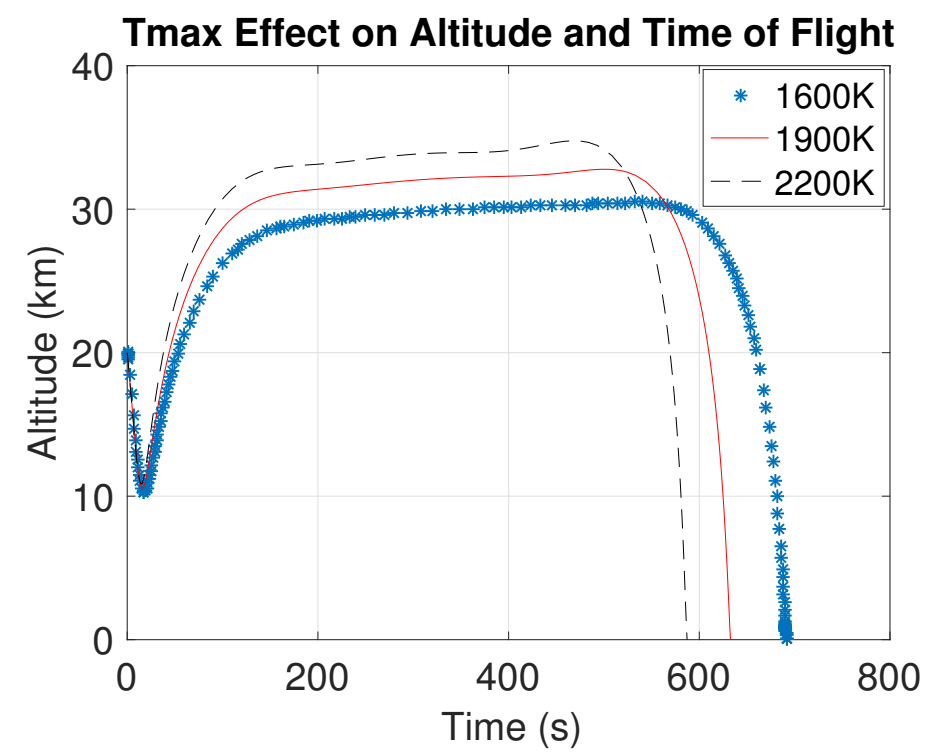

Figure 7.1. Altitude history with changing $T_{\text {Max }}$ values.

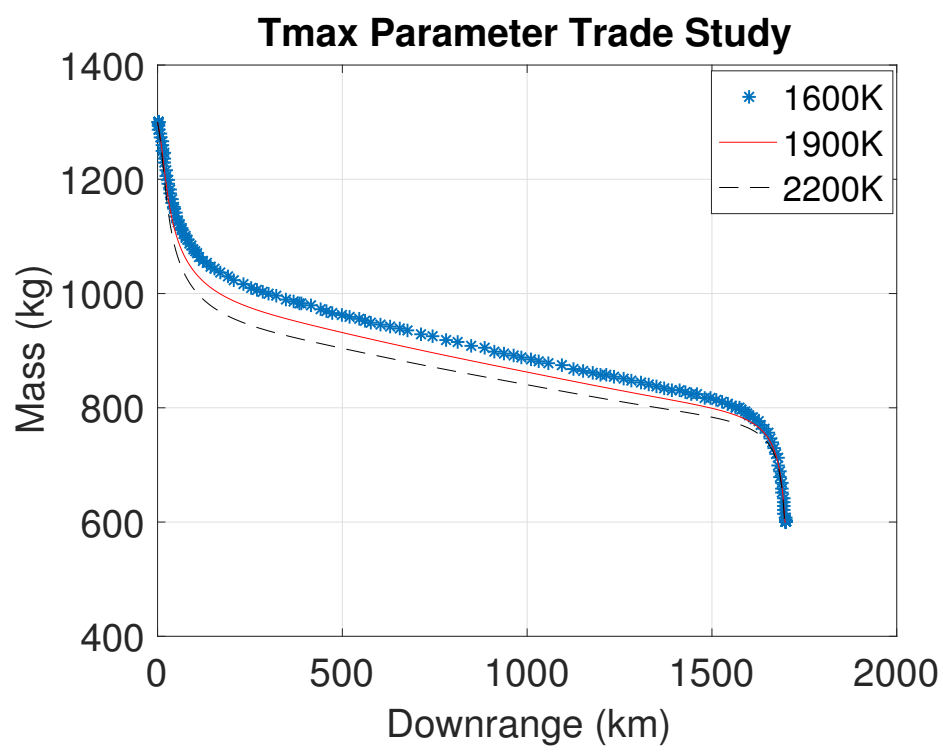

Figure 7.2. Mass by downrange study with changing $T_{\text {Max }}$ values. 


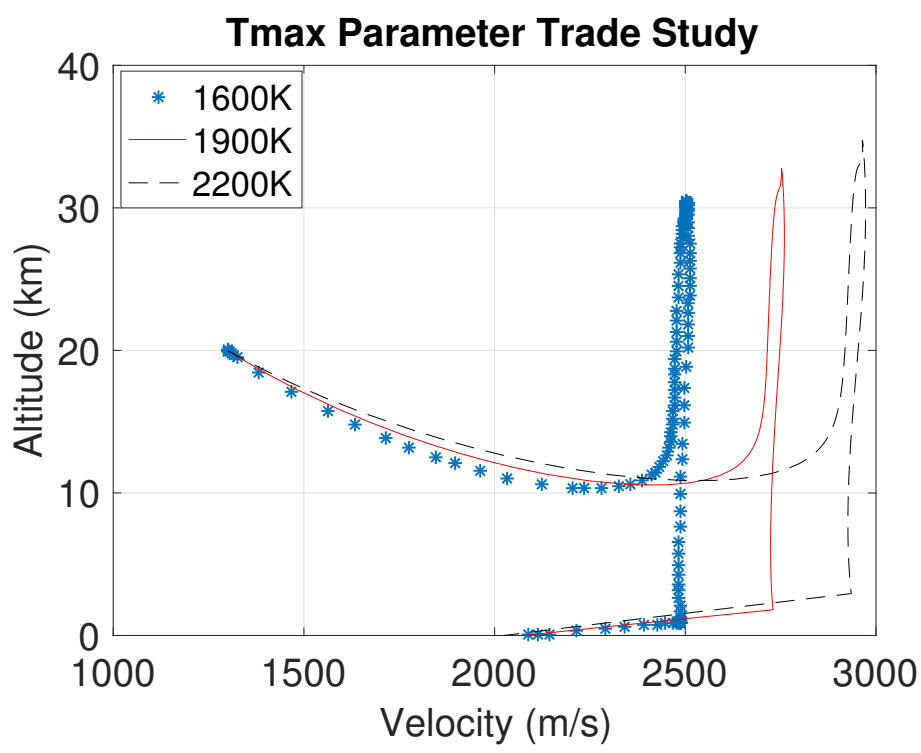

Figure 7.3. Energy plot with changing $T_{\text {Max }}$ values.

The next parameter studied was the maximum engine inlet area. The inlet area scales the thrust output linearly and this means that a change in area can have a large effect on the outcome of the mission. Observing Fig. 7.4, 7.5, and 7.6 the same trend is recognized as was seen with combustion temperature. Increasing the inlet area increases the maximum velocity and altitude while lowering the time of flight. The objective function values are recorded in Table 7.2. While the maximum inlet area can be used as a design tool to tune a vehicle toward a mission, it is less useful than improving the combustion temperature. To have the same effect on the overall mission the inlet area would need to be changed to a much larger extent.

Table 7.2. Values for scramjet engine input.

\begin{tabular}{lc} 
Amax $\left(m^{2}\right)$ & Time of Flight $(\mathrm{s})$ \\
\hline 0.2 & 742.7 \\
\hline 0.3 & 691.4 \\
\hline 0.4 & 665.5 \\
\hline
\end{tabular}




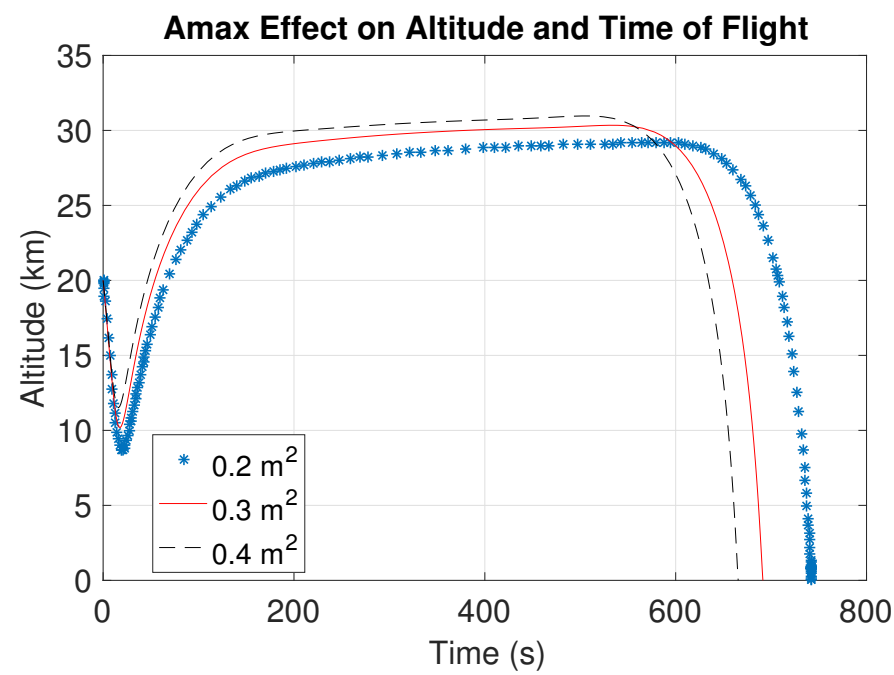

Figure 7.4. Altitude history with changing $A_{M a x}$ values.

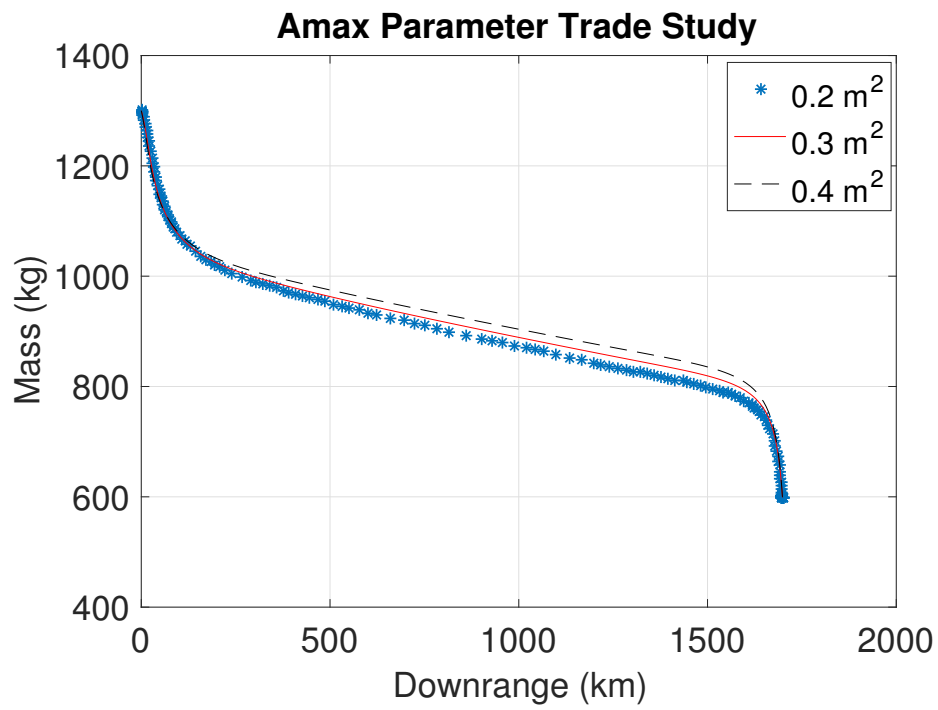

Figure 7.5. Mass by downrange with changing $A_{M a x}$ values. 


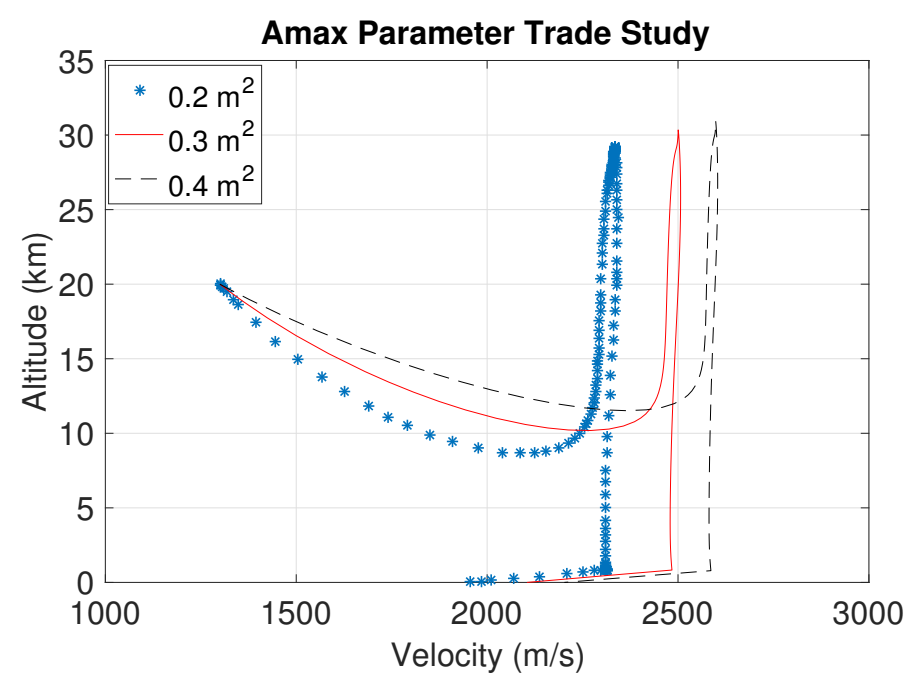

Figure 7.6. Energy plot with changing $A_{M a x}$ values.

Section 6.1 previously showed the effect that combustion Mach number had on the engine. The next trade study shows how combustion Mach number impacts the overall mission. Figures 7.7, 7.8, and 7.9 illustrate that as the engine produces more thrust and has a larger fraction of fuel flow rate per unit mass flow rate, the mission profile expands to increased maximum altitudes and velocities. $M_{c}$ of 3 versus 4 is a maximum velocity difference of approximately half a kilometer per second. The reduced drag of operating higher in the atmosphere certainly plays a role in this. The available fuel to burn is depleted at a much quicker rate. The final result of these changes is a reduction in time of flight of 129 seconds or $18.7 \%$ between the $M_{c}$ of 3 and 4 cases.

This is a significant change in the trajectory and the largest seen in this study. However, operating a scramjet combustor at a Mach number of 4 rather than 3 is a drastic change and one that is not done easily. Therefore it is not unexpected that such an improvement in engine operation would have this effect on the mission.

The final trade study conducted varied three different fuels, JP-7, JP-10, and methane. JP-7 is a common fuel used when evaluating supersonic aircraft. It's high flash point allows the fuel to be used as a heat sink for scramjet engines, cooling the 
Table 7.3. Values for scramjet engine input.

\begin{tabular}{lc}
$M_{c}$ & Time of Flight (s) \\
\hline 3 & 691.5 \\
\hline 3.5 & 621 \\
\hline 4 & 562.3
\end{tabular}

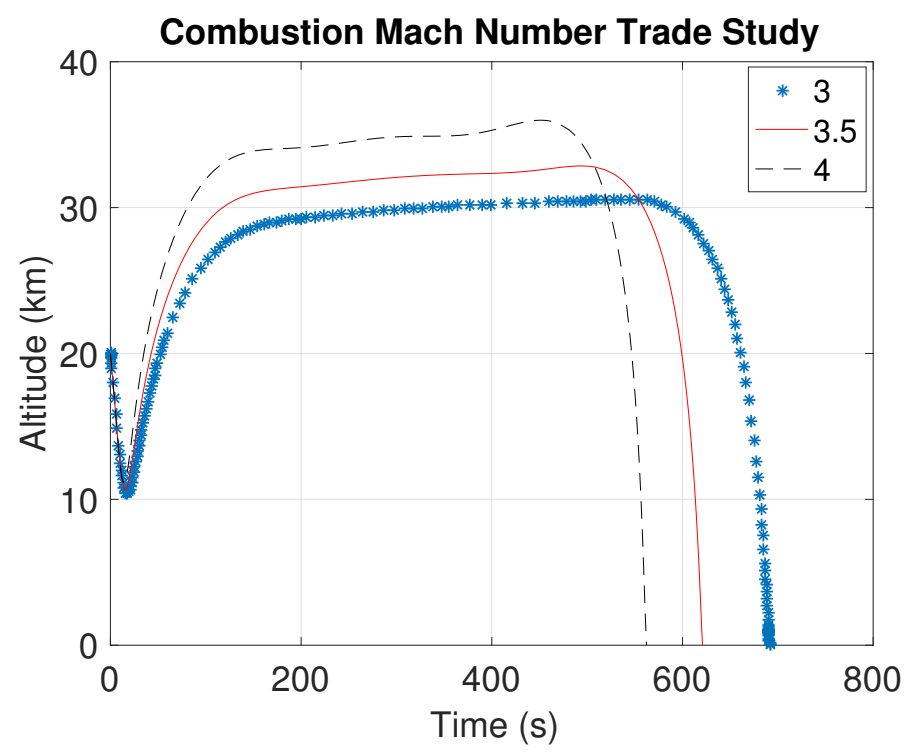

Figure 7.7. Altitude history with changing $M_{c}$ values. 


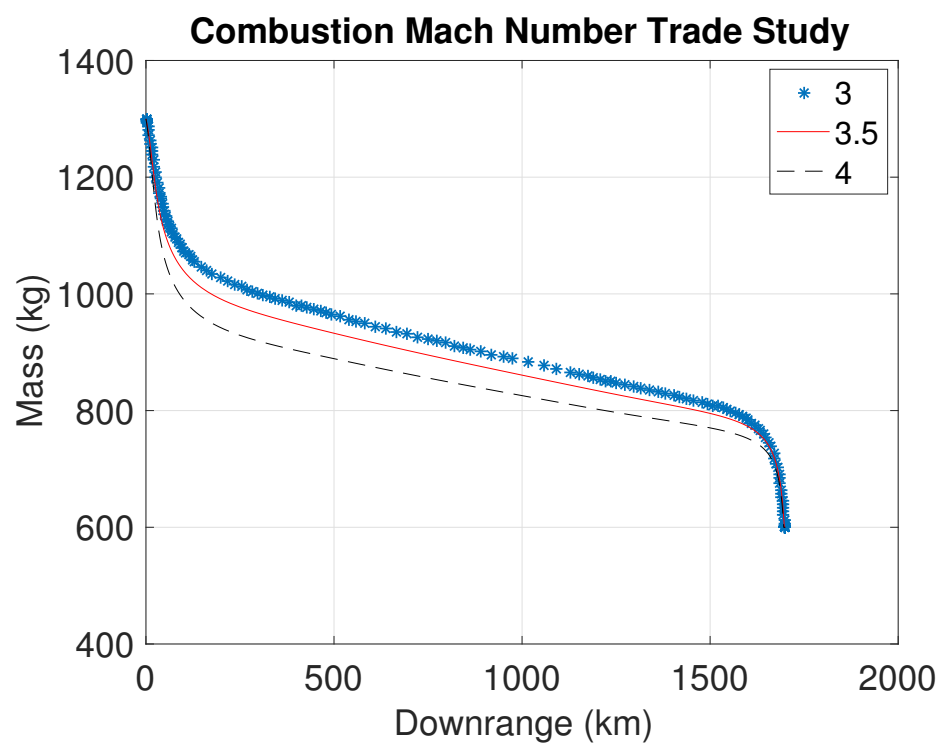

Figure 7.8. Mass by downrange with changing $M_{c}$ values.

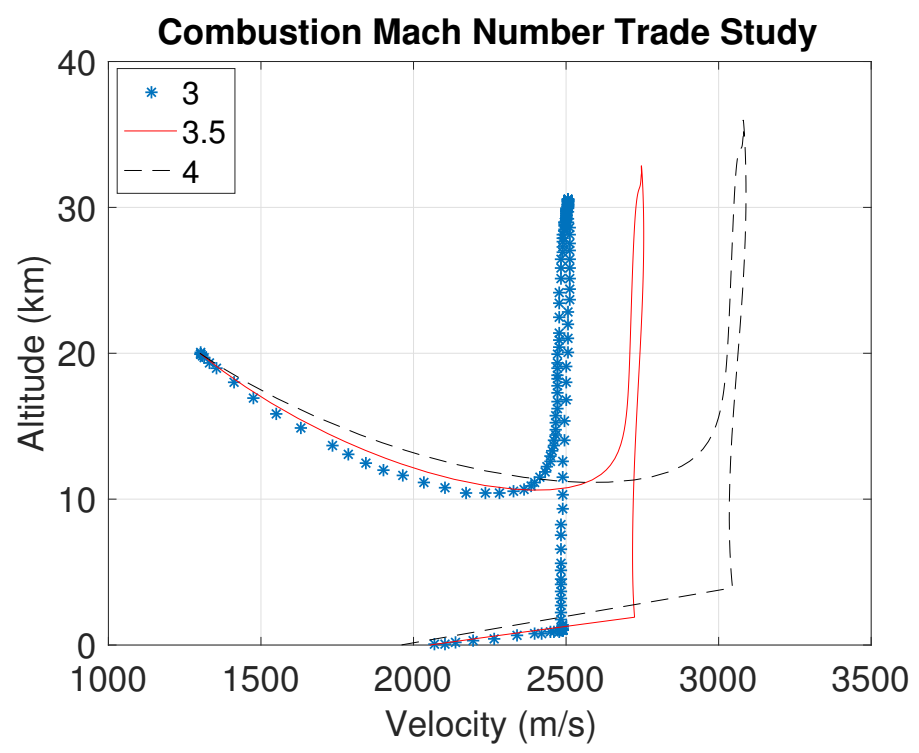

Figure 7.9. Energy plot with changing $M_{c}$ values. 
engine while cracking the fuel. JP-10 is a fuel selected for scramjets in volume limited applications due to the high density [34].

Fuel selection is accounted for in the thrust model only by the heating value, $h_{p r}$. Therefore additional benefits of a particular fuel, such as its heat sinking capability, are not considered. In Table 7.4 it is seen that the heating values of the three selected fuels are all relatively close to each other. This helps explain why the fuel selection had a relatively small effect on the overall mission. In future work, evaluating a fuel such as hydrogen, $H_{2}$, with a significantly higher heating value may have a large impact. Currently, there is nearly no difference in the time of flight between the fuels. A more comprehensive model may be needed to capture the effect of fuel selection.

Table 7.4. Values for scramjet engine input.

\begin{tabular}{lcl} 
Fuel & $h_{p r}(\mathbf{M J} / \mathbf{k g})$ & Time of Flight $(\mathbf{s})$ \\
\hline JP-10 & 42.101 & 691.8 \\
\hline JP-7 & 43.9 & 691.4 \\
\hline Methane & 50 & 690.8 \\
\hline
\end{tabular}

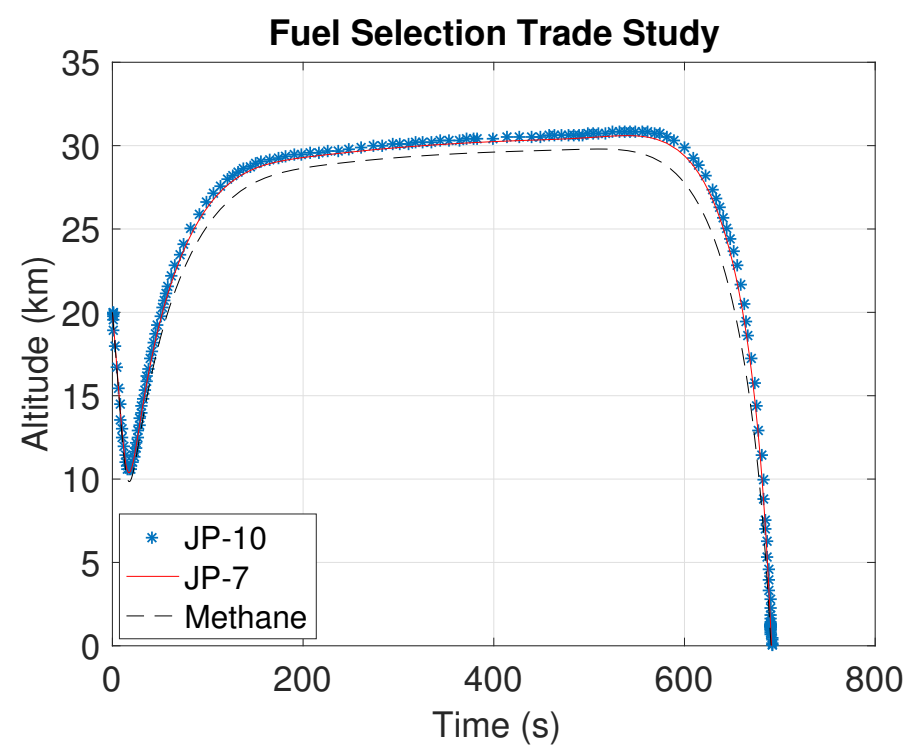

Figure 7.10. Altitude history with changing $h_{p r}$ values. 


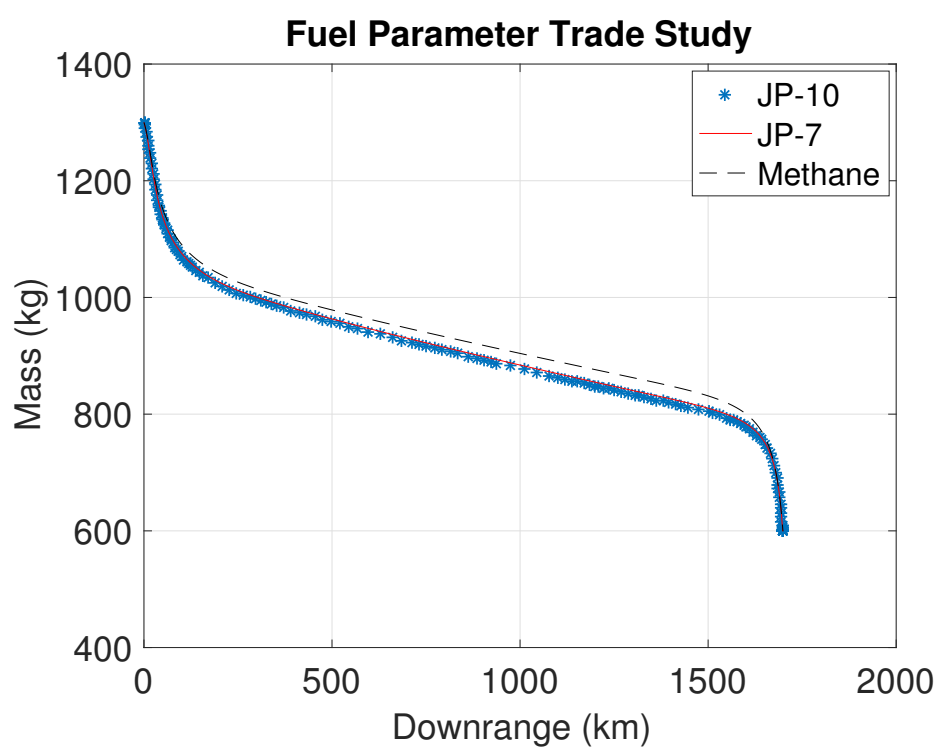

Figure 7.11. Mass by downrange with changing $h_{p r}$ values.

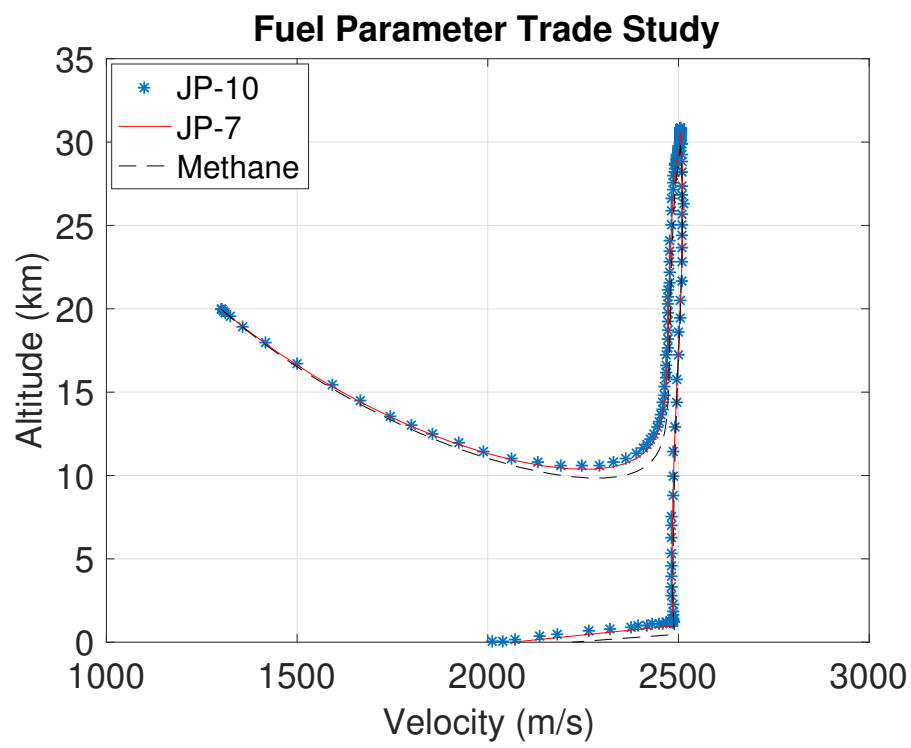

Figure 7.12. Energy plot with changing $h_{p r}$ values.

The major advantages of bridging the engine cycle analysis and trajectory optimization is the capability to conduct these various trade studies quickly. The new information attained is as follows: 
- Understanding a single parameter's effect on mission

- Contrasting performance improvements from various parameters

- Comparing multiple designs architectures

- Estimating performance of designs

Researchers will be able to study the effect parameters have on the mission scale and plan their research based on the most impactful parameters. It can also inform researchers when a parameter has reached a limit in terms of its influence on missions. Alternatively, project managers can utilize this tool to compare competing solutions against requirements early in the project, saving time and resources. With a selected design, the same project leaders can set design specific requirements for a development team. 


\section{SUMMARY}

The bridging between propulsion and trajectory/mission studies has been achieved in a way that allows for quick advancements and readily performed trade studies. Creating the solution steps and process was the primary focus of this work. Challenges regarding transcendental equations preventing analytic control laws, integrating analytic engine models, and a complex and precise solution process were overcome.

The example case shows the benefit of integrating propulsion models directly into the optimization framework. Comparison between two different vehicle architectures, the rocket and the scramjet, can be rapidly performed allowing a project manager to make informed decisions about which designs to pursue. Additionally, by adjusting parameters in the scramjet, their overall effect on the trajectory and mission at large was quickly seen. This offers the benefit of knowing what research might best improve modern missiles. From the trade studies in this thesis, combustion Mach number and maximum combustion temperature had the largest effect on reducing the time of flight. This result identifies areas of potential interest for research.

This work is just a first step in a growing mission design capability that currently contains many simplifications. However, this study has shown how to regulate power flight trajectory optimization problems with the epsilon-trig regularization method and intelligent guesses for adjoining error controls. It has also shown a capability to solve problems that the current state of the art direct solver could not. This suggests that as problem dynamics become more complicated and integrated an indirect method may be better suited to solve it. 


\section{FUTURE WORK}

The introduction of an engine cycle analysis into the trajectory optimization solver has allowed trade studies to be conducted much quicker than previous methods. Some of these capabilities were shown in Chapter 7 with combustion temperature and inlet area being readily adjusted to determine the effect on the mission. However, the complex nature of hypersonic problems meant that in this early work pieces of the problem were simplified and some complexities removed. The following is a short list of research to pursue with the completion of the present work.

- The most important task would be to implement the vehicle dynamic pressure path constraint. Adding this constraint is required for realistic trajectories. Currently, the dynamic pressures become too large for real vehicles to fly. On a similar note, the engine upper dynamic pressure bound should be reduced to a realistic value.

- Additionally, varying objective functions needs to be explored. One example of a minimum time problem was solved in this thesis, but objectives such as maximize range or impact velocity may provide increased insight into the nature of scramjet flight. These trajectories may also use the Mach bounding on the engine.

- The remaining pieces involve improving model fidelity. The first obvious improvement would regard the scramjet engine. In this work an each component of the engine was assumed ideal but as seen in many modeling works, the scramjet is complex machinery with many phenomena playing a part in its operation. Increasing complexity here would likely complicate the continuation process for attaining converged solutions, but it could also open up the possibility of more meaningful trade studies. 
- In the example problem run, the freestream Mach number varied between 4 and 8, however the aerodynamic model assumed a constant Mach of 6 . Improving the aerodynamic curve fit for more accurate results across the entire range of Mach would lead to better solutions. The difference may be particularly prominent in problems with objective functions that require flight with Mach number frequently changing.

- Finally, the exponential atmospheric model assumes a constant temperature. The loss of fidelity in the freestream temperature, $T_{0}$, can be improved by using a more accurate atmosphere model.

As the mission design capability develops and more vehicle and engine path constraints can be applied, engine parameters may eventually be moved into the optimization process and be optimized in-turn with the trajectory. Limits placed on dynamic pressure, heat loads, flight Mach numbers and more would restrict parameters from approaching infinity. This scenario represents a complete bridging of the propulsion system and vehicle trajectory. 
LIST OF REFERENCES 


\section{LIST OF REFERENCES}

[1] Ferguson, J., "TA Brief Survey of the History of the Calculus of Variations and its Applications," 2004.

[2] Ouzts, P. J., "The Joint Technology Office on Hypersonics," AIAA International Space Planes and Hypersonic Systems and Technologies Conference, Vol. 2576, 2008.

[3] Rahman, T., Hao, Z., Yongzhi, S., Younis, Y., and Kenan, Z., "Trajectory optimization of hypersonic vehicle using gauss pseudospectral method," Applied Mechanics and Materials, Vol. 110, pp. 5232-5239, 2012.

[4] Morimoto, H., and Chuang, J. CH., "Minimum-fuel trajectory along entire flight profile for a hypersonic vehicle with constraint," AIAA Guidance, Navigation, and Control Conference and Exhibit, pp. 10-12, 1998.

[5] Grant, M. J., and Bolender. M. A., "Minimum Terminal Energy Optimizations of Hypersonic Vehicles Using Indirect Methods," AIAA Atmospheric Flight Mechanics Conference, AIAA Aviation, Dallas, TX, 22-26 Jun. 2015.

[6] Longuski, J. M., Guzman, J. J., and Prussing, J. E., Optimal Control with Aerospace Applications, $1^{\text {st }}$ ed., Springer, New York, 2014.

[7] Prasanna, HM., Ghose, D., Bhat, MS., Bhattacharyya, C., and Umakant, J., "Interpolation-aware trajectory optimization for a hypersonic vehicle using nonlinear programming," AIAA Guidance, Navigation, and Control Conference and Exhibit, Vol. 6063, pp. 2005, 2005.

[8] Vinh, Nguyen X, "Integrals of the motion for optimal trajectories in atmospheric flight," AIAA Journal, Vol. 11, No. 5, 1973, pp. 700-703.

[9] Yu, Hui, and J. A. Mulder, "Arrival Trajectory Optimization on Noise Impact using Interval Analysis," AIAA Aviation Technology, Integration, and Operations Conference, Fort Worth, Texas, 13-15 Sept. 2010.

[10] Mall, K. and Grant, M. J., "Epsilon-Trig Regularization Method for Bang-Bang Optimal Control Problems," AIAA Aviation Forum and Exposition, Washington, D. C., 13-17 June, 2016 (accepted for publication)

[11] Murillo, O. J., and Ping, L., "Fast ascent trajectory optimization for hypersonic air-breathing vehicles," Proceedings of the AIAA Guidance, Navigation, and Control Conference, pp. 5-8, 2010.

[12] Roux, J. A., "Parametric Ideal Scramjet Cycle Analysis," Journal of Thermophysics and Heat Transfer, Vol. 25, No. 4, 2011, pp. 581-585. 
[13] Hank, J. M., Murphy, J. S., and Mutzman, R. C., "The X-51A Scramjet Engine Flight Demonstration Program," AIAA International Space Planes and Hypersonic Systems and Technologies Conference, Dayton, Ohio, 28 April -1 May 2008.

[14] Lu, P., "Trajectory Optimization and Guidance for a Hypersonic Vehicle," AIAA Third International Aerospace Planes Conference, Orlando, Florida, 3-5 December 1991.

[15] White, D. A., Bowers, A., Iliff, K., and Menousek, J., Flight, Propulsion, and Thermal Control of Advanced Aircraft and Hypersonic Vehicles, Handbook of Intelligent Control: Neural, Fuzzy, and Adaptive Approaches, Multiscience Press, Inc., New York, NY, 1992, pp 357-465.

[16] Bowers, A. H., and K. W. Iliff. "Generic Hypersonic Aerodynamic Model Example (GHAME) for computer simulation." NASA TM, NASA Ames-Dryden, Edwards, CA 1987.

[17] Lu, P. "Inverse dynamics approach to trajectory optimization for an aerospace plane", AIAA Journal of Guidance, Control, and Dynamics, Vol. 16, No. 4, 1993, pp. $726-732$.

[18] Curran, E. T., "Scramjet propulsion", AIAA, Vol. 189., 2001.

[19] Roberts, K. N., and Wilson, D. R. Wilson. "Analysis and design of a hypersonic scramjet engine with a transition Mach number of 4.00." Proceedings of the $4^{7}$ th AIAA Aerospace Sciences Meeting Including The New Horizons Forum and Aerospace Exposition, Orlando, Florida, 5-8 January Vol. 58, 2009.

[20] Dalle, D. J. "Interactions between Flight Dynamics and Propulsion Systems of Air-Breathing Hypersonic Vehicles." Diss. Air Force Research Laboratory, 2013.

[21] Lawden, D. F. "Optimal trajectories for space navigation" Butterworths, 1963.

[22] Lanczos, C. "The variational principles of mechanics" Courier Dover Publications Vol. 4, 1970

[23] Betts, J. T. " Methods for Optimal Control and Estimation Using Nonlinear Programming" Society for Industrial and Applied Mathematics, Vol. 19, 2010

[24] Shampine, L. F., Kierzenka J., and Reichelt M. W. "Solving boundary value problems for ordinary differential equations in MATLAB with bvp4c" Tutorial notes, pp. 437-448, 2000

[25] Herrera, M. "Galileo, Bernoulli, Leibniz and Newton around the brachistochrone problem" Rev Mexicana Fis, Vol. 40, No. 3, pp. 459-475, 1994

[26] Silva, C. J. and Trelat, E., Smooth Regularization of Bang-Bang Optimal Control Problems, IEEE, IEEE Transactions on Automatic Control, Institute of Electrical and Electronics Engineers, Vol. 55, No. 11, 2010, pp. 2488-2499.

[27] Mall, K. and Grant, M. J., Trigonomerization of Optimal Control Problems with Bounded Controls, AIAA Atmospheric Flight Mechanics Conference, Washington D.C., 13-17, June 2016 
[28] Mall, K. and Grant, M. J., Epsilon-Trig Regularization Method for Bang-Bang Optimal Control Problems, AIAA Atmospheric Flight Mechanics Conference, Washington D.C., 13-17, June 2016

[29] Strang, G., "Linear Algebra and Its Applications", 4th ed., Springer, New York, 2006.

[30] Dooloan, C. G., "Hypersonic Missile Performance and Sensitivity Analysis", Journal of Spacecraft and Rockets, Vol. 44 No. 1, Jan. - Feb, 2007.

[31] Vinh, N. X, Busemann, A., Culp, R. D. "Hypersonic and Planetary Entry Flight Mechanics," University of Michigan, 1980.

[32] Lewis, M. J., "Significance of Fuel Selection for Hypersonic Vehicle Range," Journal of Propulsion and Power, Vol. 17 No. 6, Nov. - Dec, 2001.

[33] Anthony, T. and Grant M.J. "Rapid indirect trajectory optimization on highly parallel computing architectures," Purdue University, 2014.

[34] Colket, M. B., "Scramjet Fuels Autoignition Study, "Journal of Propulsion and Power, Vol. 17 No. 2, March. - April, 2001.

[35] Smart, M., "Scramjets," In Advances on Propulsion Technology for High-Speed Aircraft, pp. 9-1 9-38, 2008.

[36] Iliff K., "From Runway to Orbit: Reflections of a NASA Engineer," 2004

[37] Smart, M.K., Hass, N.E. and Paull, A., "Flight Data Analysis of the HyShot 2 Scramjet Flight Experiment, AIAA Journal, Vol. 44, No. 10, October 2006.

[38] DuBois, R.F., Keagle, J.M. and Dudeck, C., "Science, Technology, and U.S. National Security Strategy," Center for Strategic and International Studies, Nov. 2015.

[39] Grant, M.J. and Braun, R.D., "Rapid Indirect Trajectory Optimization for Conceptual Design of Hypersonic Missions" Journal of Spacecraft and Rockets, Vol. 52, No. 1, Jan.Feb. 2015.

[40] Lu, P., Forbes, S., and Baldwin, M., "Gliding Guidance of High L/D Hypersonic Vehicles," AIAA Guidance, Navigation, and Control (GNC) Conference, Boston, MA, August 19-22, 2013. 